\title{
FUNIDAMENTAL CONSIDERATIONS FOR THE FINITE ELEMENT ANALYSIS OF SHELL STRUCTURES
}

\author{
D. Chapelle $\Varangle$ and K. J. Bathe $\ddagger$ \\ $\ddagger$ I ahoratoire Central des Ponts et Chaussées, IMMR 113 ICCC/CNRS, 2 allée Kepler, 77420 Champs- \\ sur-Marne, France \\ §Massachusetts Institute of Technology, Cambridge, MA 02139, USA
}

(Received 17 December 1996; accepted 30 July 1997)

\begin{abstract}
The objective in this paper is to present fundamental considerations regarding the finite element analysis of shell structures. First, we review some well-known results regarding the asymptotic behaviour of a shell mathematical model. When the thickness becomes small, the shell behaviour falls into one of two dramatically different categories; namely, the membrane-dominated and bending-dominated cases. The shell geometry and boundary conditions decide into which category the shell structure falls, and a seemingly small change in these conditions can result into a change of category and hence into a dramatically different shell behaviour.

An effective finite element scheme should be applicable to both categories of shell behaviour and the rate of convergence in either case should be optimal and independent of the shell thickness. Such a finite element scheme is difficult to achieve but it is important that existing procedures be analysed and measured with due regard to these considerations. To this end, we present theoretical considerations and we propose appropriate shell analysis test cases for numerical evaluations. (C) 1997 Elsevier Science Ltd
\end{abstract}

\section{INTRODUCTION}

Numerous shell firite elements have been proposed and yet there is a consensus that there are still difficulties in analysing, general shell structures. Also, it is difficult to identify which shell elements are the most effective elements currently available, and how to arrive at more efficient shell analysis procedures.

Shell structures may be called the prima donnas of structures. Their behaviour is difficult to analyse and can be somewhat unpredictable in that apparently small changes of geometry or support conditions can result into a totally different response. Finite element discretizations of shell structures therefore can also show sensitivity to the geometry and support conditions. When such sensitivity arises, certain aspects in the discretization are frequently blamed without reference to the fundamental reasons as to why there might be a different shell structural behaviour.

Considering the presently available finite element schemes for shell analysis, we frequently find that the specific procedures work quite well for certain for shell problems but, but example due to numerical locking [1], do not work well for other problems. Clearly, it is this numerical unreliability and the insufficient knowledge of the actual physical sensi-

§Present address: IINRIA, BP105, 78153 Le Chesnay Cedex, France. tivity of the shell structures that together can cloud the understanding of the analysis results.

Our objective in this paper is to give insight and understanding in the finite element analysis of shell structures. We aim to accomplish this goal by first recalling the crucial and well-known fact that the behaviour of a shell structure is dramatically different depending on whether it is a membrane-dominated or bending-dominated structure, see for example $[2,3,4]$ and the references therein. This discussion leads us to point out how we can identify into which category a shell structure falls and that a structure may, with seemingly only small changes in geometry or boundary conditions, change from one to the other category.

An effective finite element discretization must of course reflect this change in fundamental behaviour and should perform well in both membrane- and bending-dominated situations. Indeed, ideally, the error in the solution of the mathematical shell model for a given finite element interpolation should be independent of the shell thickness and reflect an optimal convergence behaviour for any stress situation (membrane- or bending-dominated) encountered.

However, mathematical analyses of the available finite elements regarding these requirements are at present hardly available. Therefore, it is prudent to use effective numerical tests that identify in how far a specific finite element fulfills the requirements and 


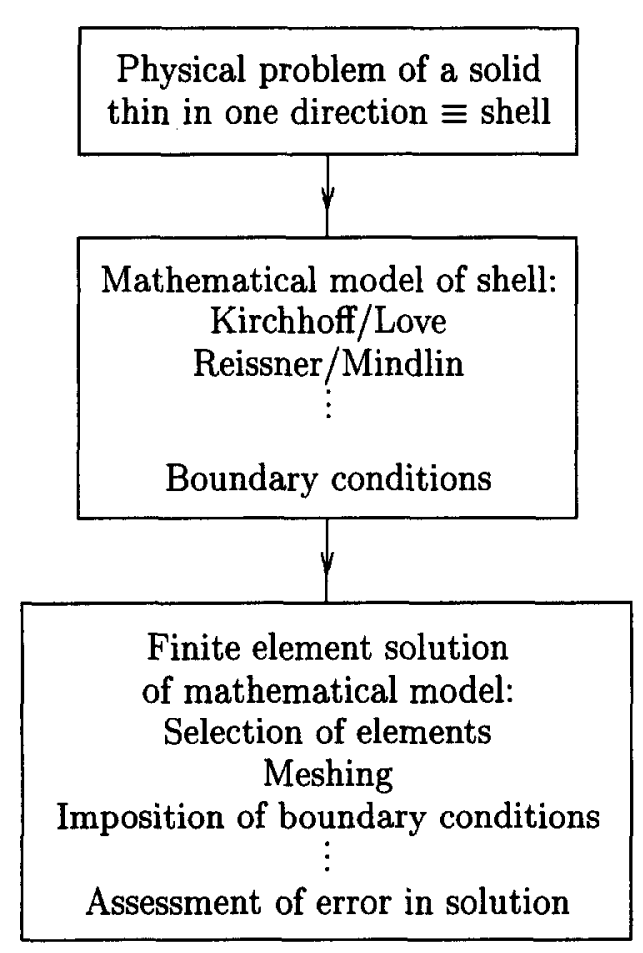

Fig. 1. Finite element analysis of shell problems.

thus represents a valuable general tool for the analysis of shell structures. In this paper we discuss, using basic theoretical considerations, earlier proposed numerical tests, and propose additional new test cases.

Figure 1 summarizes the finite element solution process of a shell structure. The first step is to choose a shell mathematical model. Frequently used models are based on the Reissner-Mindlin approximations but lower- and higher-order shell mathematical models can also be employed and indeed, a fully three-dimensional solution of the thin structure may be sought. The selected mathematical model is then in the second step solved using finite element procedures, and here we require the use of effective finite clements that for any shell geometry, thickness and boundary conditions yield an accurate solution. This finite element solution is to be performed quite automatically and should also give error measures that indicate how accurately the mathematical model was solved [1]. In the discussion to follow, we should keep in mind the distinction between the shell mathematical model (whose exact solution we seek), and the numerical solution of that model using finite element procedures [1].

While we consider in this paper two-dimensional shell models, as mentioned in Fig. 1, the analysis and conclusions are also applicable, to a large extent, to the degenerate three-dimensional isoparametric finite element formulations for general shell analyses. In these isoparametric formulations, the kinematic and stress assumptions used in shell the- ories are directly incorporated into interpolations to provide the finite element procedure [1].

\section{ASYMPTOTIC BEHAVIOUR OF SHELL MATHEMATICAL MODELS}

While, in principle, we proceed in the finite element analysis of a shell structure as we do in the analysis of a solid, there are specific difficulties due to the thinness of the shell. These difficulties become apparent when studying the asymptotic behaviour of a shell mathematical model when the thickness becomes very small (and in the limit approaches zero). The purpose of this section is to review some fundamental facts regarding shell mathematical models in order to establish a framework for studying the finite element solution of these models.

\subsection{General framework}

We consider a shell to be a solid medium geometrically defined by a mid-surface immersed in the three-dimensional space, and a parameter representing the thickness of the medium lying around this surface. For simplicity of discussion, we henceforth consider shells of constant thickness, but the same considerations are also applicable to varying thickness shell structures, and indeed also to composite shells. The behaviours of the shell models are governed by a system of equations involving unknowns that are defined on the mid-surface (namely displacements and rotations). These shell models are considered as given prior to the analysis performed here and we therefore do not discuss their justifications. For mathematical justifications of some shell models obtained by asymptotic analysis techniques where the starting point is the continuous medium subjected to laws of three-dimensional elasticity, refer for example to Refs [5-8]. As is usual, for each formulation that we consider here we refer to authors who established the models earlier and used mechanical arguments. Also, we restrict our investigation to linear models. These indeed already suffer from the numerical difficulties which motivate our approach, and attempting to treat them first in this simplified framework seems to be a natural strategy.

In what follows, we assume that the shell midsurface, $\mathcal{S}$, is defined by a single chart, which is a one-to-one smooth mapping from $\bar{\Omega}$ into $R^{3}$, where $\Omega$ denotes an open domain of $R^{2}$ called "reference domain" and thus $\mathcal{S}=(\phi \bar{\Omega})$, sec Fig. 2 ( $\overline{\mathbf{\Omega}}$ denotes the closure of $\Omega$, i.e. the union of $\Omega$ and its boundary $\partial \Omega$ ). We now briefly recall the classical definitions and notation of differential geometry that we need for our purposes, see $\operatorname{Refs}[9,10]$ for more details. We use the Einstein convention on the summation of repeated indices, their values ranging in $\{1,2\}$. Let the covariant base of the tangential plane be defined by 


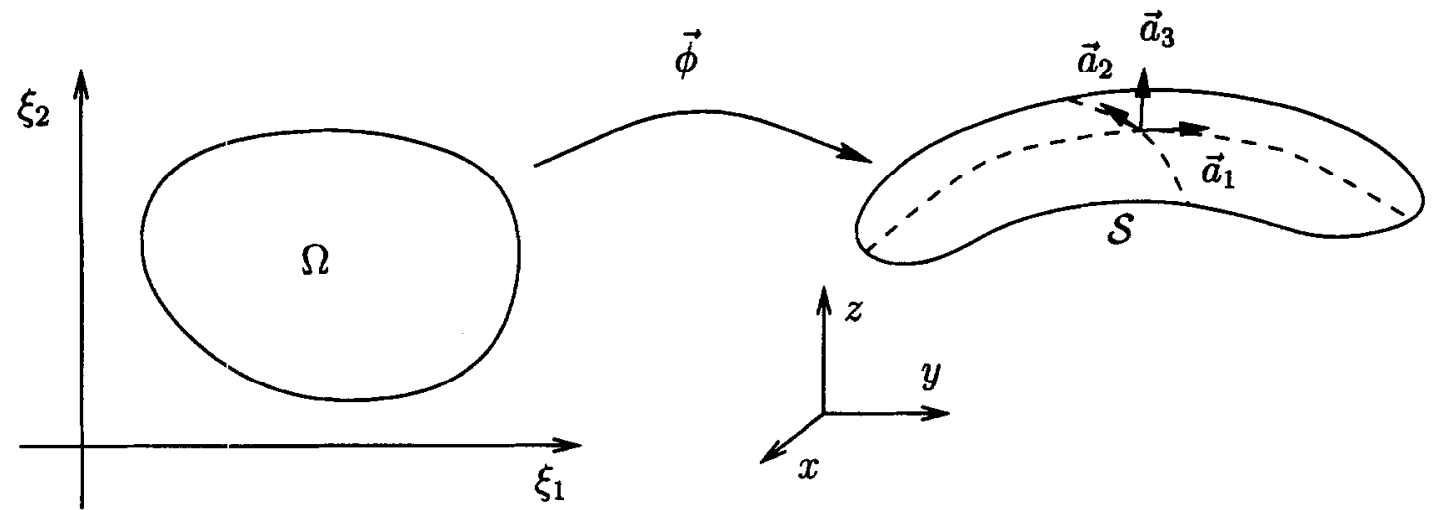

Fig. 2. Definition of the shell surface by a chart.

$$
a_{\alpha} \stackrel{\operatorname{def}}{=} \frac{\partial \phi\left(\xi_{1}, \xi_{2}\right)}{\partial \xi_{\alpha}},
$$

with the contravariant base given by

$$
\mathbf{a}_{\alpha} \cdot \mathbf{a}^{\beta}=\delta_{\alpha}^{\beta},
$$

where $\delta$ denotes the Kronecker symbol. The unit normal vector is

$$
\mathbf{a}_{3}=\frac{\mathbf{a}_{1} \times \mathbf{a}_{2}}{\left\|\mathbf{a}_{1} \times \mathbf{a}_{2}\right\|} .
$$

The first fundamental form of the surface is given by

$$
a_{\alpha \beta} \stackrel{\text { def }}{=} \mathbf{a}_{\alpha} \cdot \mathbf{a}_{\beta}
$$

or alternatively in contravariant form by

$$
a^{\alpha \beta} \stackrel{\text { def }}{=} \mathbf{a}^{\alpha} \cdot \mathbf{a}^{\beta} .
$$

The second fundamental form is defined by

$$
b_{\alpha \beta} \stackrel{\text { def }}{=} \mathbf{a}_{3} \cdot \mathbf{a}_{\alpha, \beta},
$$

and the third fundamental form by

$$
c_{\alpha \beta} \stackrel{\text { def }}{=} b_{\alpha}^{\lambda} b_{\lambda \beta},
$$

where we recall that

$$
b_{\alpha}^{\beta} \stackrel{\text { def }}{=} a^{\beta \lambda} b_{\lambda \alpha} .
$$

The following symbol appears in surface measures:

$$
a \stackrel{\text { def }}{=}\left\|\mathbf{a}_{1} \times \mathbf{a}_{2}\right\|^{2}=a_{11} a_{22}-\left(a_{12}\right)^{2},
$$

and indeed we denote

$$
d S \stackrel{\text { def }}{=} \sqrt{a} d \xi_{1} d \xi_{2}
$$

Finally, we denote the covariant differentiation by a vertical bar (like in " $v_{\alpha \mid \beta}$ ", see Ref. [10]).

\subsection{Shell mathematical models considered}

The generic form for the variational formulations of the shell mathematical models that we consider is

$$
t^{3} A(U ; V)+t D(U ; V)=G(V), \quad \forall V \in \mathcal{U},
$$

where $U$ is the unknown solution field which we call "displacements" even though some components may be of a different nature (namely rotations), and $\mathcal{U}$ is the set of "admissible displacements" taking into account the appropriate functional spaces and the boundary conditions. The symbol $t$ denotes the shell thickness. In the sequel we study how the properties of the model change when $t$ varies, and particularly when $t$ becomes very small compared to the other characteristic dimensions of the system. $A$ and $D$ are bilinear forms whose expressions depend on the model considered, but not on the parameter $t$. Finally, the right-hand side $G(V)$ represents the work of external forces for the virtual displacement $V$.

The models that we examine here fall into two main categories: models of the Kirchhoff-Love type, and models of the Reissner-Mindlin type, socalled by analogy with plate models.

In Reissner-Mindlin type models, the unknowns are the displacement of the mid-surface (a vector in $R^{3}$ denoted by $\mathbf{u}$ ), and the rotation of the vector orthogonal to the mid-surface (a surface tensor of order 1 which we denote by $\underline{\theta}$ [11]. The Naghdi formulation can be written as [9]:

Find $(\mathbf{u}, \underline{\theta})$ in $\mathcal{U}^{\mathrm{RM}}$ such that

$$
\begin{gathered}
t^{3} A^{\mathrm{RM}}(\mathbf{u}, \underline{\theta} ; \mathbf{v}, \underline{\eta})+t D^{\mathrm{RM}}(\mathbf{u}, \underline{\theta} ; \mathbf{v}, \underline{\eta})=G(\mathbf{v}), \\
\forall(\mathbf{v}, \eta) \in \mathcal{U}^{\mathrm{RM}} .
\end{gathered}
$$

Here

$$
A^{\mathrm{RM}}(\mathbf{u}, \underline{\theta} ; \mathbf{v}, \underline{\eta}) \stackrel{\text { def }}{=} \int_{\Omega} \frac{E^{\alpha \beta \lambda \mu}}{12} \chi_{\alpha \beta}(\mathbf{u}, \underline{\theta}) \chi_{\lambda \mu \mu}(\mathbf{v}, \underline{\eta}) \mathrm{d} S
$$

which can be interpreted as the internal work of the 
bending stresses. The bending strain operator $\chi$ is expressed as

$$
\chi_{\lambda \mu}(\mathbf{v}, \underline{\eta}) \stackrel{\text { def }}{=} \frac{1}{2}\left[\eta_{\lambda \mid \mu}+\eta_{\mu \mid \lambda}-b_{\lambda}^{\alpha} v_{\alpha \mid \mu}-b_{\mu}^{\alpha} v_{\alpha \mid \lambda}\right]+c_{\lambda \mu} v_{3} .
$$

The above fourth-order tensor is

$$
E^{\alpha \beta \lambda \mu} \stackrel{\text { def }}{=} \frac{E}{2(1+v)}\left[a^{\alpha \lambda} a^{\beta \mu}+a^{\alpha \mu} a^{\beta \lambda}+\frac{2 v}{1-v} a^{\alpha \beta} a^{\lambda \mu}\right],
$$

where $E$ and v, respectively, denote Young's modulus and Poisson's ratio for the material. The second bilinear form $D^{\mathrm{RM}}$ can be written as the sum of a membrane term $D^{\mathrm{m}}$ and a shear term $D^{\mathrm{s}}$ :

$$
\begin{array}{r}
D^{\mathrm{m}}(\mathbf{u}, \mathbf{v}) \stackrel{\text { def }}{=} \int_{\Omega} E^{\alpha \beta \lambda \mu} \gamma_{\alpha \beta}(\mathbf{u}) \gamma_{\lambda \mu}(\mathbf{v}) \mathrm{d} S \\
D^{\mathbf{s}}(\mathbf{u}, \underline{\theta} ; \mathbf{v}, \underline{\eta}) \stackrel{\text { def }}{=} \int_{\Omega} J^{\alpha \beta} \varphi_{\alpha}(\mathbf{u}, \underline{\theta}) \varphi_{\beta}(\mathbf{v}, \underline{\eta}) \mathrm{d} S
\end{array}
$$

with

$$
\begin{aligned}
& \gamma_{\lambda \mu}(\mathbf{v}) \stackrel{\text { def }}{=} \frac{1}{2}\left(v_{\lambda \mid \mu}+v_{\mu \mid \lambda}\right)-b_{\lambda \mu} v_{3}, \\
& \varphi_{\beta}(\mathbf{v}, \underline{\eta}) \stackrel{\text { def }}{=} v_{3, \beta}+b_{\beta}^{\lambda} v_{\lambda}+\eta_{\beta}, \\
& J^{\alpha \beta} \stackrel{\text { def }}{=} \frac{E a^{\alpha \beta}}{2(1+v)} .
\end{aligned}
$$

Finally, the effect of external forces appears through

$$
G(\mathrm{v}) \stackrel{\text { def }}{=} \int_{\Omega} \mathrm{p} \cdot \mathrm{vd} S
$$

where $\mathbf{p}$ is the distributed force over the mid-surface $\mathcal{S}$.

In Kirchhoff-Love type models, the only unknown is the displacement vector $u$. These models are based on stronger kinematic assumptions than the Reissner-Mindlin type models, in that the rotation is inferred from the displacement, typically by assuming that normal vectors remain orthogonal to the mid-surface in the deformed configuration. Consequently, shear terms are not included in the formulation. As an example, we use Koiter's model [12], whose expression in the linear framework is:

Find $\mathbf{u}$ in $\mathcal{U}^{\mathrm{K}}$ such that

$$
t^{3} A^{\mathrm{K}}(\mathbf{u} ; \mathbf{v})+t D^{\mathrm{K}}(\mathbf{u} ; \mathbf{v})=G(\mathbf{v}), \quad \forall \mathbf{v} \in \mathcal{U}^{\mathrm{K}},
$$

with

$$
A^{\mathrm{K}}(\mathbf{u} ; \mathbf{v}) \stackrel{\text { def }}{=} \int_{\Omega} \frac{E^{\alpha \beta \lambda \mu}}{12} \bar{\rho}_{\alpha \beta}(\mathbf{u}) \bar{\rho}_{\lambda \mu}(\mathbf{v}) \mathrm{d} S
$$

where

$$
\bar{\rho}_{\lambda \mu}(\mathbf{v}) \stackrel{\text { def }}{=} v_{3 \mid \lambda \mu}-c_{\lambda \mu} v_{3}+b_{\lambda \mid \mu}^{\alpha} v_{\alpha}+b_{\lambda}^{\alpha} v_{\alpha \mid \mu}+b_{\mu}^{\alpha} v_{\alpha \mid \lambda} .
$$

Again, $A^{\mathrm{K}}$ can be interpreted as a bending term. $D^{\mathrm{K}}$ is identical to the membrane part of the previous formulation:

$$
D^{\mathrm{K}}(\mathbf{u} ; \mathbf{v}) \stackrel{\text { def }}{=} D^{\mathrm{m}}(\mathbf{u} ; \mathbf{v})
$$

and the right-hand side is as before. We know that both formulations (2) and (3) are well-posed mathematical problems, provided we specify the appropriate functional framework:

$$
\begin{aligned}
& \mathcal{U}^{\mathrm{RM}} \stackrel{\text { def }}{=}\left\{V=(\mathbf{v}, \underline{\eta}) \mid \mathrm{v} \in\left[H^{1}(\Omega)\right]^{3}, \underline{\eta} \in\left[H^{1}(\Omega)\right]^{2}\right\} \cap \mathcal{C L}, \\
& \mathcal{U}^{\mathrm{K}} \stackrel{\text { def }}{=}\left\{V=\mathrm{v} \mid\left(v_{1}, v_{2}\right) \in\left[H^{1}(\Omega)\right]^{2}, v_{3} \in H^{2}(\Omega)\right\} \cap \mathcal{C L},
\end{aligned}
$$

where $\mathcal{C L}$ symbolically denotes the essential boundary conditions imposed on the admissible displacements. Of course, these boundary conditions are supposed to be compatible with the nature of each functional space, and sufficient to prevent rigid body motions. Then these formulations can be shown to satisfy the proper continuity and ellipticity properties $[13,14]$, viz.

$$
c_{\mathrm{t}}\|V\|_{\mathcal{U}}^{2} \leq t^{3} A(V ; V)+t D(V ; V) \leq C_{\mathrm{t}}\|V\|_{\mathcal{U}}^{2}, \quad \forall V \in \mathcal{U},
$$

where $\|\cdot\|_{\mathcal{U}}$ denotes the norm used for $\mathcal{U}$, according to the nature of the Sobolev spaces involved. Thus, recalling the definition of the $L^{2}, H^{1}$ and $H^{2}$ norms:

$$
\begin{aligned}
& \|\tau\|_{L^{2}} \stackrel{\text { def }}{=}\left[\int_{\Omega} \tau^{2} \mathrm{~d} \Omega\right]^{1 / 2}, \\
& \|\tau\|_{H^{1}} \stackrel{\text { def }}{=}\left\{\|\tau\|_{L^{2}}^{2}+\sum_{\alpha=1,2}\left\|\frac{\partial \tau}{\partial \xi_{\alpha}}\right\|_{L^{2}}^{2}\right\}^{1 / 2}, \\
& \|\tau\|_{H^{2}} \stackrel{\text { def }}{=}\left\{\|\tau\|_{H^{1}}^{2}+\sum_{1 \leq \alpha \leq \beta \leq 2}\left\|\frac{\partial^{2} \tau}{\partial \xi_{\alpha} \partial \xi_{\beta}}\right\|_{L^{2}}^{2}\right\}^{1 / 2},
\end{aligned}
$$

the norms used for the two models described above are

$$
\|\mathbf{v}, \underline{\eta}\|_{\mathcal{U}^{\mathrm{RM}}} \stackrel{\text { def }}{=}\left\{\sum_{i=1,2,3}\left\|\mathrm{v}_{i}\right\|_{H^{1}}^{2}+\sum_{\alpha=1,2}\left\|\eta_{\alpha}\right\|_{H^{1}}^{2}\right\}^{1 / 2}
$$

and

$$
\|\mathbf{v}\|_{\mathcal{U}^{\mathrm{k}}} \stackrel{\text { def }}{=}\left\{\sum_{\alpha=1,2}\left\|\mathrm{v}_{\alpha}\right\|_{H^{1}}^{2}+\left\|\mathrm{v}_{3}\right\|_{H^{2}}^{2}\right\}^{1 / 2}
$$

Equation (4) guarantees the well-posedness of the variational problem. Note, however, that the ellipticity and continuity constants $\left(c_{t}\right.$ and $C_{t}$, respectively) are $t$-dependent.

\subsection{Shell geometries considered}

In the sequel, we often categorize surface geometries according to the nature of their curvature tensor. We recall that a surface is called elliptic, parabolic or hyperbolic according to whether the Gaussian curvature $K$, given by

$$
K \stackrel{\text { def }}{=} \frac{b_{11} b_{22}-\left(b_{12}\right)^{2}}{a}
$$

is positive, zero or negative, respectively. For example, a part of an ellipsoid is an elliptic surface; a cylinder, a cone, and developable surfaces in gen- 


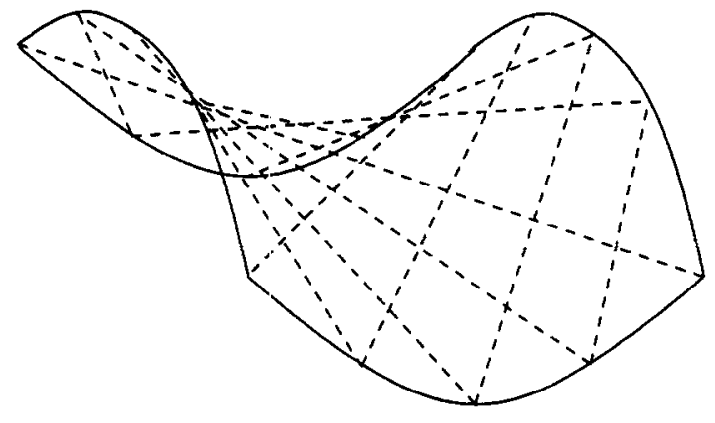

Fig. 3. Hyperbolic paraboloid and its asymptotic lines.

eral are all parabolic surfaces; a hyperbolic paraboloid of equation $z=x y$ (see Fig. 3) is a hyperbolic surface.

Of course, general surfaces need not be of uniform nature in this respect, but this distinction can always be made pointwise. It is a fundamental distinction because, as we show in Section 2.5 , shells of different geometric nature behave very differently when their thickness becomes small. For this reason, surfaces of uniform geometric nature (i.e. cverywherc elliptic, or parabolic, or hyperbolic), such as most examples that we consider in the discussions to follow, should not be thought of as restrictive cases bur, on the contrary, as essential ingredients that determine the behaviour of more general structures cobtained by assembling surfaces of various types.

\subsection{Asymptotic behelviour}

Implicit in the concept of a "shell" is the idea that the thickness is "small" compared to the other two dimensions. In practice, it is not unusual to deal with structures whose thickness is smaller by several orders of magnitude, in which case the shell is said to be "thin" (consider, for example, the shell body of a motor car). Considering the role of $t$ in Equation (1), with weights varying from 1 to $t^{2}$ in front of the different terms on the left-hand side, it is reasonable to wonder in what way the properties of the model are affected when this parameter becomes small. Likewise, it is important to know whether the model converges, in some sense, towards a "limit model" when $t$ tends to zero, so that this possibly simpler model can be used and analysed instead of the original one when $t$ is sufficiently small. As already mentioned, our ultimate goal is to investigate the influence of the thickness on the convergence of numerical methods, in order to be able to detect difficulties such as numerical locking. We first concentrate on the asymptotic behaviour of the mathematical models, whereas the issues arising in the finite element solutions are specifically dealt with in Section 3.

In an asymptotic: analysis, one particular subspace of $\mathcal{U}$ takes on a crucial role (see for example $\operatorname{Refs}[5,7,15,16]$, namely

$$
\mathcal{U}_{0} \stackrel{\text { def }}{=}\{V \in \mathcal{U} \mid D(V ; V)=0\} .
$$

For the model in Equation (3), this space is

$$
\mathcal{U}_{0}^{\mathrm{K}} \stackrel{\text { def }}{=}\left\{\mathbf{v} \in \mathcal{U}^{\mathrm{k}} \mid \gamma_{\alpha \beta}(\mathbf{v})=0, \quad \alpha, \beta=1,2\right\},
$$

and thus $\mathcal{U}_{0}^{\mathrm{K}}$ is the subspace of displacements with vanishing membrane strains. For the model in Equation (2), we have

$$
\mathcal{U}_{0}^{\mathrm{RM}} \stackrel{\text { def }}{=}\left\{\begin{array}{lcc}
(\mathbf{v}, \underline{\eta}) \in \mathcal{U}^{\mathrm{RM}} \mid & \gamma_{\alpha \beta}(\mathbf{v})=0, & \alpha, \beta=1,2 \\
& \phi_{\alpha}(\mathbf{v}, \underline{\eta})=0, & \alpha=1,2
\end{array}\right\},
$$

and both membrane and shear strains must vanish. In both cases $\mathcal{U}_{0}$ contains the displacements for which only the bending strains are non-zero, and hence it is the subspace of pure bending displacements. Note that, for the Reissner-Mindlin model, the condition of vanishing shear strains is equivalent to the following relations

$$
\eta_{\alpha}=-\left(v_{3, \alpha}+b_{\alpha}^{\lambda} v_{\lambda}\right),
$$

which provide an explicit expression of $\eta$ as a function of $\mathbf{v}$. Therefore, the governing conditions for $\mathcal{U}_{0}^{\mathrm{RM}}$ are also those of vanishing membrane strains, like for $\mathcal{U}_{0}^{\mathrm{K}}$. For this reason, $\mathcal{U}_{0}$ is also called, in general, the subspace of inextensional displacements.

Unlike what happens for simpler structures such as beams and plates, we observe that the conditions which define $\mathcal{U}_{0}$ make up an exactly determined set of equations (i.e. we have as many equations as unknowns). This reveals an essential specificity of shells, which is that a situation where

$$
\mathcal{U}_{0}=\{0\}
$$

is possible. Such a situation is designated to be a case of "inhibited pure bending". We proceed to show that the asymptotic behaviour of a shell model is highly dependent on whether or not pure bending is inhibited. Examples of shells for which Equation (7) is and is not applicable are given and discussed in Section 2.5.

2.4.1. The case of non-inhibited pure bending. The case of non-inhibited pure bending is of course, for example, encountered in the analysis of flat shells, that is, in plate formulations. However, we consider the more general case of a shell. We know that, for the solution of Equation (1) to remain both bounded and non-vanishing, it must be assumed that the right-hand side is of the form

$$
G(V)=t^{3} F(V),
$$

where $F$ is a linear form independent of $t$, see for example Refs $[15,17,18]$. In other words, we can say that the stiffness of the structure varies like $t^{3}$. For each value of $t$, the problem to be solved is then: 
Find $U_{t} \in \mathcal{U}$ such that

$$
A(U t ; V)+\frac{1}{t^{2}} D\left(U_{t} ; V\right)=F(V), \quad \forall V \in \mathcal{U} .
$$

When $t$ is very small, this problem can be interpreted as a penalized form of the following problem of optimization under constraints:

Find $U_{0} \in \mathcal{U}_{0}$ such that

$$
A\left(U_{0} ; V\right)=F(V), \quad \forall V \in \mathcal{U}_{0} .
$$

This new problem is well posed since it is the restriction to a closed subspace of the elliptic optimization problem, Equation (9). Furthermore, the following convergence result can be established [19] (see also $\operatorname{Refs}[20,21]$ ).

Proposition 1. When $t$ tends to zero, the solution $U_{t}$ of Equation (9) converges to the solution $U_{0}$ of Equation (10) for the norm of $\mathcal{U}$. Additionally, we have

$$
\lim _{t \rightarrow 0} \frac{1}{t^{2}} D\left(U_{i} ; U_{t}\right)=0
$$

Equation (11) means that in the ReissnerMindlin type models the membrane and shear energies tend to zero with $t$, and in the Kirchhoff-Love type models, of course, only the membrane energy tends to zero since the shear energy is always zero. This explains why shells with non-inhibited pure bending are sometimes also called "bending dominated" structures.

2.4.2. The case of inhibited pure bending. We recall that, in the case of inhibited pure bending, $\mathcal{U}_{0}=\{0\}$ and therefore

$$
D(V ; V)>0, \quad \forall V \in \mathcal{U} \backslash\{0\} .
$$

Hence, $D(V ; V)^{1 / 2}$ provides a norm on $\mathcal{U}$. In fact, this norm can be defined on a space larger than $\mathcal{U}$, denoted by $\mathcal{V}$, which consists of all possible displacements $V$ for which $D(V ; V)$ is bounded. Namely, we only enforce in $\mathcal{V}$ the regularity requirements corresponding to the membrane part of the total energy. $\mathcal{V}$ is of course less regular than $\mathcal{U}$, and its exact nature depends on the shell considered and on the boundary conditions imposed. For Koiter's model, for instance, if the surface is uniformly elliptic, sufficiently smooth, and clamped along its whole boundary, we can show that pure bending is inhibited and that $\mathcal{V}$ is $\left[H_{0}^{1}(\Omega)\right]^{2} \times L^{2}(\Omega)[22,23]$.

A proper scaling for the right-hand side is then

$$
G(V)=t F(V)
$$

i.e. the stiffness is now of the order of $t$, see for example Refs $[7,16]$. The problem sequence becomes:

Find $U_{t} \in \mathcal{U}$ such that

$$
D\left(U_{t} ; V\right)+t^{2} A\left(U_{t} ; V\right)=F(V), \quad \forall V \in \mathcal{U} .
$$

This is a classical singular perturbation of an elliptic problem [24]. We can define the limit problem:

Find $U_{l} \in \mathcal{V}$ such that

$$
D\left(U_{t} ; V\right)=F(V), \quad \forall V \in \mathcal{V} .
$$

This problem is well posed provided the right-hand side can be made sense of, that is $F$ must be in the dual space of $\mathcal{V}$. An equivalent condition for that is

$$
|F(V)| \leq C D(V ; V)^{1 / 2}, \quad \forall V \in \mathcal{U},
$$

where $C$ is a constant. Note that $F$ being in the dual space of $\mathcal{V}$ is not at all guaranteed since, $\mathcal{V}$ being larger than $\mathcal{U}, \mathcal{V}^{\prime}$ is smaller than $\mathcal{U}^{\prime}$. If the condition holds, then the singularly-perturbed problem converges, as expressed in the following proposition $[24,16]$.

Proposition 2. If $F \in \mathcal{V}^{\prime}$, the solution $U_{t}$ of Equation (13) converges to the solution $U_{l}$ of Equation (14) when $t$ tends to zero for the norm $D(V ; V)^{1 / 2}$. Furthermore, it holds that

$$
\lim _{t \rightarrow 0} t^{2} A\left(U_{t} ; U_{t}\right)=0 \text {. }
$$

Hence, Equation (16) shows that the bending energy in this case becomes negligible compared to the other terms when $t$ tends to zero, as opposed to what happens for non-inhibited shells. Another significant difference between the two asymptotic behaviours is that, for an inhibited shell, a loss of regularity occurs in the transition from $\mathcal{U}$ to $\mathcal{V}$, unlike for non-inhibited shells from $\mathcal{U}$ to $\mathcal{U}_{0}$. In certain cases, this loss of regularity is not without serious consequences. For instance, for a uniformly elliptic shell described by the Koiter model with displacements fixed on part of the boundary only, pure bending can be shown to be inhibited but the space $\mathcal{V}$ is not, mathematically speaking, a distribution space [25]. This implies that there exist some indefinitely differentiable functions that do not belong to the dual of $\mathcal{V}$, and hence lead to ill-posed membrane problems when used as loadings. As a matter of fact, it is well known in engineering practice that any reasonable loading applied on such a structure gives a "physically unstable" membrane problem.

\subsection{Analysis of the subspace of pure bending displa- cements}

We have already emphasized the critical role of the subspace of pure bending displacements, depending on whether or not it is restricted to $\{0\}$. We also observed that the governing conditions which characterize this subspace are the zero membrane strains, viz.

$$
\gamma_{\alpha \beta}(\mathbf{v})=0, \quad \alpha, \beta=1,2 .
$$

A remarkable property of this system of differential equations, proved in Ref. [16], is that the nature of the differential equations (elliptic, parabolic or 
hyperbolic) is the same as the geometric nature of the surface at the point in consideration. This allows us to non-exhaustively review several instances for which it is possible to tell whether or not pure bending is inhibited. Obviously this property also depends on the boundary conditions which we therefore need to take into consideration. We now examine shells whose mid-surface is of uniform nature (everywhere elliptic, or parabolic, or hyperbolic).

2.5.1. Elliptic surfaces. For a sufficiently smooth uniformly elliptic surface, for instance part of an ellipsoid, imposing zero displacements on a part of the boundary of non-zero measure is sufficient to inhibit pure bending displacements [25]. As already mentioned, if this part extends to the whole boundary, then there is a limit problem which retains sufficient regularity for the solution. However, with less support on the structure, the problem is always ill-posed. By contrast, if no boundary condition is imposed except for preventing rigid body motions, then there exists pure bending displacements [26].

2.5.2. Hyperbolic surfaces. For a hyperbolic surface (see for instance the hyperbolic paraboloid in Fig. 3 with the lattice of straight asymptotic lines), we infer that system (17) is itself of hyperbolic nature. Moreover, its characteristics are the asymptotic lines of the surface [25]. Therefore, the subspace of pure bending displacements is highly dependent on the boundary conditions which together with Equation (17) define a Cauchy problem that in general is well posed.

In order to make the analysis of the problem simpler, it is valuable to use the coordinate system defined by the asymptotic lines of the surface. This is always possible when the surface is sufficiently smooth. System (17) then becomes:

$$
\left.\begin{array}{c}
v_{1 \mid 1}=0 \\
v_{2 \mid 2}=0 \\
\frac{1}{2}\left(v_{1 \mid 2 !}+v_{2 \mid 1}\right)=b_{12} v_{3}
\end{array}\right\}
$$

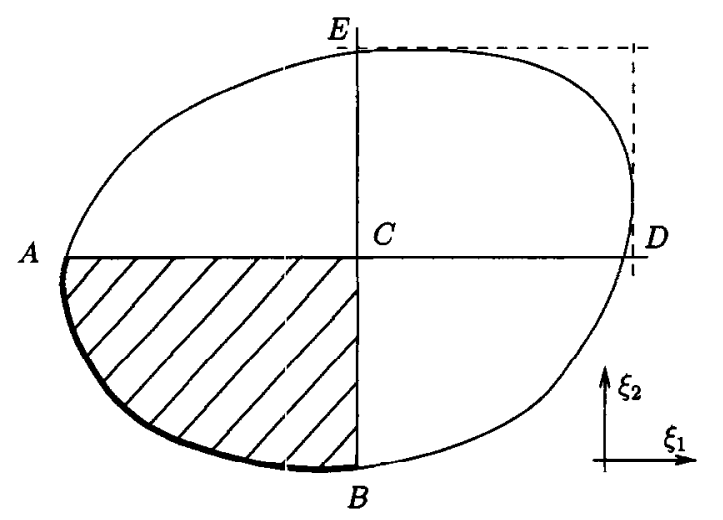

Fig. 4. Hyperbolic susface in the asymptotic coordinates. with $b_{12}>0$, and therefore the third equation gives $v_{3}$ explicitly in terms of $v_{1}$ and $v_{2}$.

As an example, we display in Fig. 4 the reference domain of a hyperbolic surface in this particular coordinate system. Suppose that $v_{1}$ and $v_{2}$ are fixed on the part $(A B)$ of the boundary. Then the Cauchy problem is well posed in the triangle $(A B C)$ where therefore all displacements are zero. However, outside of this region there exist non-zero displacements which satisfy Equation (18). For instance, we can arbitrarily set $v_{1}$ on the line $(C E)$ and $v_{2}$ on the line $(C D)$, and this determines the value of the fields over the whole domain.

Thus, unlike with elliptic surfaces, hyperbolic surfaces appear to easily lead to non-inhibited situations, provided that boundary conditions are imposed on a sufficiently restricted part of the boundary.

2.5.3. Parabolic surfaces. Parabolic surfaces, i.e. surfaces of zero Gaussian curvature (cylinders, cones, etc.), can be thought of as a narrow category between the two above-mentioned major types. In practice, however, these geometric shapes are by far the most employed. Therefore, the analysis of their mechanical behaviour is of particular importance.

For the sake of simplicity, we focus on the cylindrical case (the analysis for a cone would be similar). We use the natural coordinate system (defined by the rulings and the cross-sections, see Fig. 5) in which all Christoffel symbols are zero and the covariant base is orthonormal. System (17) then reads

$$
\begin{aligned}
v_{1,1} & =0 \\
v_{2,2} & =b_{22} v_{3} \\
v_{1,2}+v_{2,1} & =0
\end{aligned}
$$

As is easily seen, if we prescribe the displacements on some part of a cross-section, the entire corresponding band gets inhibited (see Fig. 5 where the section is fixed on part $(A B)$ ). By contrast, if boundary conditions are only imposed along rulings (i.e. straight lines parallel to the axis of the cylinder), pure bending displacements remain possible:

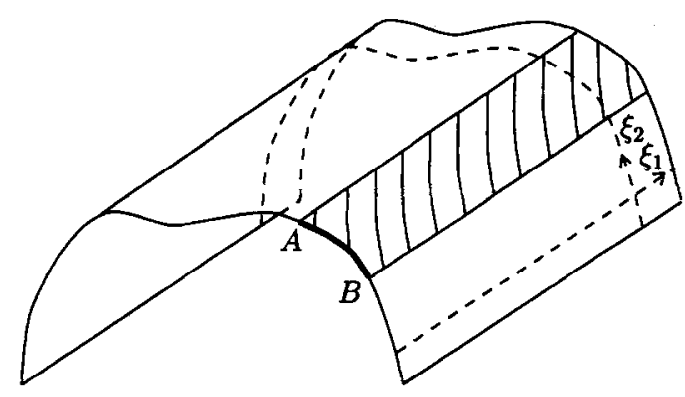

Fig. 5. Natural coordinates and inhibited region for a cylinder. 
$v_{1}$ and $v_{2}$ can in fact be set arbitrarily along any cross-section (subject to satisfying the boundary conditions) and a complete field of pure bending displacements is thus fully determined. For instance arch-like displacements, i.e. those in which all crosssections undergo identical displacements in their own planes and according to a pure bending arch mode belong to the pure bending subspace.

\subsection{Influence of the loading}

In the previous discussion, the governing factor in the analysis of the asymptotic behaviour of a shell model was the content of the subspace of pure bending displacements, $\mathcal{U}_{0}$, which depends on the geometry of the surface and the boundary conditions only. As regards the loading, we only mentioned the regularity issues that determine whether or not a membrane problem is well posed (through Condition (15)). Such a minor influence of the loading may seem surprising, in particular when recalling that certain load distributions can be found that do not activate the pure bending displacements, and hence cannot possibly give rise to bending-dominated asymptotic behaviour. However, an analysis shows that even small perturbations to such load distributions result in significant displacements when $t$ is small, and hence, in practice, the load distribution (apart from the regularity issues) need not be considered. In this section, we present this analysis and an illuminating example for which a closed form solution can be obtained.

2.6.1. Effect of loadings that do not activate the pure bending displacements. We consider for Equation (1) a right-hand side of the form

$$
G(V)=t^{\rho} F(V) \text {, }
$$

where $\rho$ is a scaling exponent to be determined, and $F$ is such that

$$
F(V)=0, \quad \forall V \in \mathcal{U}_{0}
$$

Of course, we suppose that $\mathcal{U}_{0} \neq\{0\}$, i.e. that pure bending is not inhibited. Thus, the loading considered here does not activate the (non-trivial) pure bending displacements.

The general philosophy of an asymptotic analysis consists in finding the right exponent $\rho$ such that a limit problem can be defined when $t$ tends to zero, the solution of this limit problem being non-zero. In this specific case, if we choose the scaling $\rho=3$ as indicated in Section 2.4.1, Proposition 1 ensures that convergence occurs to the solution of Equation (10), which is zero because of Equation (21). Therefore this scaling is unable to capture the relevant asymptotic behaviour. In the next proposition, we show that, assuming that Equation (15) is satisfied (note that this is possible here because Equation (21) holds, even though pure bending is non-inhibited), the asymptotic behaviour is of the membrane type. The problem sequence in consideration is thus:

Find $\tilde{U}_{t} \in \mathcal{U}$ such that

$$
D\left(\tilde{U}_{t} ; V\right)+t^{2} A\left(\tilde{U}_{t} ; V\right)=F(V), \quad \forall V \in \mathcal{U} .
$$

We first need a preliminary result, stated in the following lemma.

Lemma 1. $\mathcal{U}$ can be decomposed into the direct sum

$$
\mathcal{U}=\mathcal{U}_{0} \oplus \mathcal{U}_{c}
$$

where $\mathcal{U}_{c}$ is the subspace of $\mathcal{U}$ uniquely defined by

$$
A(U ; V)=0, \quad \forall(U, V) \in \mathcal{U}_{0} \times \mathcal{U}_{c} .
$$

Proof: for any fixed $t>0$, Equation (4) expresses that $\left(t D(V ; V)+t^{3} A(V ; V)\right)^{1 / 2}$ defines a norm that is equivalent to the norm $\|V\|_{\mathcal{U}}$. Define $\mathcal{U}_{c}$ as the subspace orthogonal to $\mathcal{U}_{0}$ for the dot product associated with this new norm. We have

$$
t D(U ; V)+t^{3} A(U ; V)=0, \quad \forall(U, V) \in \mathcal{U}_{0} \times \mathcal{U}_{c}
$$

Note that

$$
D(U, W)=0, \quad \forall(U, W) \in \mathcal{U}_{0} \times \mathcal{U},
$$

since, by the Cauchy-Schwarz inequality, $|D(U ; W)|$ $\leqslant D(U ; U)^{1 / 2} D(W ; W)^{1 / 2}=0$. Hence, Equation (25) is equivalent to Equation (24), which shows that $\mathcal{U}_{c}$ is defined independently of $t$.

We observe that, since $\mathcal{U}_{0} \cap \mathcal{U}_{c}=\{0\}, D(V ; V)^{1 / 2}$ defines a norm on $\mathcal{U}_{c}$, and we can consider a space $\mathcal{V}_{c}$, defined as the extension of $\mathcal{U}_{c}$ for this norm exactly as $\mathcal{V}$ was defined from $\mathcal{U}$ in Section 2.4.2. We now state the convergence result.

Proposition 3. For any $t>0$, the solution $\tilde{U}_{t}$ of Problem (22) is in $\mathcal{U}_{c}$. Moreover, assuming that Equation (15) holds, when $t$ tends to zero, $\tilde{U}_{t}$ converges, for the norm $D(V ; V)^{1 / 2}$, to $\tilde{U}_{l}$ defined as the unique element of $V_{c}$ that satisties

$$
D\left(\tilde{U}_{l} ; V\right)=F(V), \quad \forall V \in \mathcal{V}_{c} .
$$

In addition, we have

$$
\lim _{t \rightarrow 0} t^{2} A\left(\tilde{U}_{t} ; \tilde{U}_{t}\right)=0
$$

Proof: for any $t>0$, decompose $\tilde{U}_{t}$ into

$$
\tilde{U}_{t}=\tilde{U}_{0 t}+\tilde{U}_{c t},
$$

with $\tilde{U}_{0 t} \in \mathcal{U}_{0}$ and $\tilde{U}_{c t} \in \mathcal{U}_{c}$. Then, choosing $V \in \mathcal{U}_{0}$ in Equation (22) and using Equations (21), (24) and (26), we obtain

$$
A\left(\tilde{U}_{0 t} ; V\right)=0, \quad \forall V \in \mathcal{U}_{0},
$$

hence $\tilde{U}_{0 t}=0$, since $A$ is coercive on $\mathcal{U}_{0}$ (take $V \in \mathcal{U}_{0}$ in Equation (4)). Therefore $\tilde{U}_{t}=\tilde{U}_{c t} \in \mathcal{U}_{c}$. Taking now $V \in \mathcal{U}_{c}$ in Equation (22), we get 


$$
D\left(\tilde{U}_{t} ; V\right)+t^{2} A\left(\tilde{U}_{t} ; V\right)=F(V), \quad \forall V \in \mathcal{U}_{c},
$$

and this problem is of the exact same type as Equation (13). Since Equation (15) holds, the above properties are a direct application of Proposition 2.

Therefore, when the loading does not activate the pure bending displacements, as expressed by Equation (21), the alsymptotic behaviour of the solution is formally similar to that in the case of inhibited pure bending. Namely, assuming that Equation (15) is satisfied, the solution of the shell problem converges to the solution of a membrane problem, provided that the loading is scaled according to Equation (12) (i.e. the membrane type scaling).

However, to understand the general behaviour of a shell structure we of course need to consider various loading distributions, and in particular also the effect of perturbations on the loading used above. Such perturbed loading can be represented by substituting $F+\bar{F}$ for $F$ in Equation (22), assuming that $F$ satisfies Equation (21) but not $\bar{F}$, and that the amplitude of $\bar{F}$ is "small" compared to that of $F$. Note that $\bar{F}$, although activating the bending deformations, is assumed to correspond to a value of $\rho=1$ in Equation (20). Then, due to the linearity of the problem, the new solution is $\tilde{U}_{t}+\bar{U}_{t} / t^{2}$, where $\tilde{U}_{t}$ is the solution of Equation (22) and $\bar{U}_{s}$ the solution of:

$$
A\left(\bar{U}_{t} ; V\right)+\frac{1}{t^{2}} D\left(\bar{U}_{t} ; V\right)=\bar{F}(V), \quad \forall V \in \mathcal{U},
$$

which is a penalized problem similar to Fquation (9). Here, both $\tilde{U}_{t}$ and $\bar{U}_{t}$ are convergent, according to Prop. 3 and Prop. 1, respectively. These convergence behaviours correspond to two different norms, therefore we cannot directly compare the terms $\tilde{U}_{t}$ and $\bar{U}_{t} / t^{2}$. We can, however, compare the asymptotic orders of their respective energies, i.e.

$$
\mathcal{E}(V) \stackrel{\text { def }}{=} t)(V ; V)+t^{3} A(V ; V),
$$

for $V=\tilde{U}_{t}$ and for $V=\bar{U}_{t} / t^{2}$. For $\tilde{U}_{t}$, according to Prop. 3, the dominant part of the energy is $t D\left(\tilde{U}_{t} ; \tilde{U}_{t}\right)$, and $D\left(\tilde{U}_{t}, \tilde{U}_{t}\right)$ converges to a finite value. By contrast, for $\bar{U}_{t} / t^{2}$, Prop. 1 implies that the dominant term is $t^{-1} A\left(\bar{U}_{i} ; \bar{U}_{t}\right)$, where $A\left(\bar{U}_{t} ; \bar{U}_{t}\right)$ converges to a finite value. Thus, even though the amplitude of the perturbation is small, the effect of this perturbation becomes dominant when $t$ is sufficiently small, with a relative amplification factor proportional to $1 / t^{2}$.

We proceed to illustrate this discussion by an example.

2.6.2. A demonstrative solution: the case of an infnitely long cylinder loaded by internal pressure. We consider an infinitely long circular cylinder of radius $R$, with the natural coordinate system defined in
Section 2.5.3. We use the Naghdi shell formulation, Equation (2). The cylinder is loaded by a (non-constant) internal pressure independent of the $\xi_{1}$ coordinate. Therefore, the solution is independent of $\xi_{1}$ and, for symmetry reasons,

$$
u_{1}=\theta_{1}=0 \text {. }
$$

For certain choices of the pressure distribution, we can derive closed-form solutions of the problem by solving simple systems of linear equations. This will allow us to illustrate our above discussions. We first define a non-dimensional parameter characteristic of the thickness:

$$
d \stackrel{\text { def }}{=} \frac{t}{R},
$$

and three constants:

$$
\beta_{1} \stackrel{\text { def }}{=} \frac{1}{12\left(1-v^{2}\right)}, \quad \beta_{2} \stackrel{\text { def }}{=} \frac{1}{1-v^{2}}, \quad \beta_{3} \stackrel{\text { def }}{=} \frac{1}{2(1+\nu)} .
$$

If a constant pressure is applied on the cylinder, pure bending displacements are obviously not activated. We therefore consider the following righthand side:

$$
G(V)=d \int_{\Omega} \bar{f} v_{3} \mathrm{~d} S,
$$

where ${ }^{-} f$ is a constant. Note that this is consistent with the membrane type scaling. We obtain the following values for the displacements:

$$
\begin{aligned}
& u_{2}=\theta_{2}=0, \\
& u_{3}=\frac{f R}{E} \frac{1}{\beta_{2}+\beta_{1} d^{2}}
\end{aligned}
$$

For a slice of unit length, the energy values corresponding to the bending and membrane parts are, respectively,

$$
\begin{aligned}
& \mathcal{E}_{\mathrm{b}}=\pi \beta_{1} \frac{f^{2} R^{2}}{E} \frac{d^{3}}{\left(\beta_{2}+\beta_{1} d^{2}\right)^{2}}, \\
& \mathcal{E}_{\mathrm{m}}=\pi \beta_{2} \frac{f^{2} R^{2}}{E} \frac{d}{\left(\beta_{2}+\beta_{1} d^{2}\right)^{2}},
\end{aligned}
$$

and the shear energy is zero.

Suppose now that the constant pressure above is perturbed by the term

$$
\bar{G}(V)=d \int_{\Omega} \bar{f} \cos \left(2 \xi_{2} / R\right) v_{3} \mathrm{~d} S,
$$

where $\bar{f}$ is a constant much smaller than $f$. We can compute the solution corresponding to this perturbation analytically. We get, for the displacements

$$
\begin{aligned}
& \bar{u}_{2}=-\frac{\bar{f} R}{E}\left(\frac{1}{18 \beta_{1}} d^{-2}+\frac{5}{9 \beta_{2}}+\frac{2}{9 \beta_{3}}\right) \sin \left(2 \xi_{2} / R\right), \\
& \bar{u}_{3}=\frac{\bar{f} R}{E}\left(\frac{1}{9 \beta_{1}} d^{-2}+\frac{4}{9 \beta_{2}}+\frac{4}{9 \beta_{3}}\right) \cos \left(2 \xi_{2} / R\right),
\end{aligned}
$$




$$
\vec{\theta}_{2}=\frac{\bar{f}}{E}\left(\frac{1}{6 \beta_{1}} d^{-2}+\frac{1}{3 \beta_{2}}\right) \sin \left(2 \xi_{2} / R\right),
$$

and for the bending, membrane and shear energies, respectively,

$$
\begin{aligned}
\mathcal{E}_{\mathrm{b}} & =\frac{\pi}{18 \beta_{1}} \frac{\bar{f}^{2} R^{2}}{E} d^{-1}, \\
\mathcal{E}_{\mathrm{m}} & =\frac{2 \pi}{9 \beta_{2}} \frac{\bar{f}^{2} R^{2}}{E} d, \\
\mathcal{E} s & =\frac{2 \pi}{9 \beta_{3}} \frac{\bar{f}^{2} R^{2}}{R} d .
\end{aligned}
$$

Of course, these results are completely consistent with the earlier given theoretical discussions. Here, even though $\tilde{f}$ is much smaller than $f$, the effect of the perturbation is dominant in the displacements themselves when $t$ is smaller than $\sqrt{R \bar{f} / f}$, and in the energy also when $t$ is smaller than $R \bar{f} \mid f$.

\section{ASYMPTOTIC ANALYSIS OF FINITE ELEMENT METHODS FOR THIN SHELLS}

Considering a shell with a fixed, known a priori thickness, ellipticity results show that any conforming finite element method applied to Equation (1) admits an error estimate which is bounded by the interpolation estimate and a constant that depends on the shell thickness $[13,14]$. For a thin shell, this constant is large and the error in the solution can be unacceptable. Supposing that the exact solution of the model retains "reasonable properties" (e.g. bounded and regular), it is natural to seek a finite element scheme that provides optimal results for all values of $t$ (and indeed independent of $t$ ). Such a finite element scheme is difficult to develop as testified by numerous famous examples of numerical locking exhibited in the finite element solution of thin structures. Thus, an asymptotic analysis of the finite element methods themselves is necessary. We now consider this analysis separately for the inhibited and non-inhibited cases.

\subsection{Non-inhibited shells}

We mentioned that, in the case of non-inhibited shells, the sequence of problems (9) can be interpreted as a penalized form of an optimization problem under constraints. It is precisely in this context that numerical locking can arise; namely this phenomenon appears when the discrete displacements, of the space denoted by $\mathcal{U}^{h}$, are unable to properly satisfy the constraint [1]. In the most serious instances, we indeed have

$$
\mathcal{U}^{h} \cap \mathcal{U}_{0}=\{0\} .
$$

Let us denote by $U_{t}^{h}$ the finite element solution, i.e. the element of $\mathcal{U}^{h}$ such that

$$
A\left(U_{t}^{h} ; V\right)+\frac{1}{t^{2}} D\left(U_{t}^{h} ; V\right)=F(V), \quad \forall V \in \mathcal{U}^{h} .
$$

We then infer from Proposition 1, in this particular case of a finite dimensional space, that

$$
\lim _{t \rightarrow 0} U_{t}^{h}=0 .
$$

By contrast

$$
U_{0}=\lim _{t \rightarrow 0} U_{t} \neq 0 .
$$

Here, the meaning of the term "numerical locking" becomes clear: when $t$ tends to zero the numerical scheme provides results that appruach zero displacements, whereas the exact solution itself remains non-vanishing.

For non-inhibited shells, we proceed to show that, with conforming finite element discretizations and certain shell structures, Equation (44) in fact holds. We consider the case of a hyperbolic surface for which, as seen above, a non-inhibited situation is common.

Proposition 4. Consider a regular hyperbolic shell fixed on some part of its boundary, and a finite element scheme, in the framework of Equation (45), in which the displacement components $v_{1}, v_{2}$ and $v_{3}$ are approximated by continuous piecewise-polynomial functions. Assume that no edge of the elements in the mesh is part of an asymptotic line. Then Equation (44) holds.

Proof: we will show that, for any $\left(v_{1}, v_{2}, v_{3}\right) \in \mathcal{U}^{h} \cap \mathcal{U}_{0}$, if $v_{1}$ and $v_{2}$ equal zero on an edge of any element of the mesh, then $v_{1}, v_{2}$ and $v_{3}$ are identically zero over the whole element. This being granted, the result is immediately obtained by "propagating" the zero displacements from the boundary conditions to cover the whole domain.

Consider an arbitrary element of the mesh, with $v_{1}$ and $v_{2}$ set to zero on any of its edges, then we have two possibilities:

(a) Either the lattice of characteristic curves originating from this edge covers the element completely (like in Fig. 6a) and the property directly follows.

(b) Or the element is only partially covered (Fig. 6b), but in this case we still have $v_{1}=v_{2}=v_{3}=0$ on a part of non-zero area of the 28 element. Recalling that the displacements are

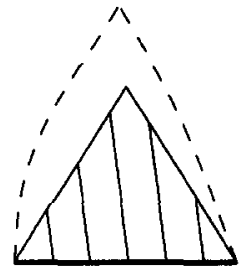

a)

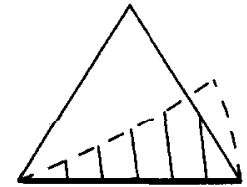

b)
Fig. 6. Characteristics and inhibited region for an element. 
given by polynomial functions, they necessarily equal zero over the whole element.

To make the statement of this property simpler, we assumed that no single element edge corresponds to an asymptotic curve. It is obvious, however, that this result carries over to any case in which the propagation technique can be applied. By contrast, if a mesh happens to be entirely supported by asymptotic curves, one should of course not deduce that locking is overcome. This simply means that locking cannot be mathematically proven to arise by the same arguments.

Note further that this result reveals a significant difference between numerical methods for shells and for other structures. For beams and plates, it is indeed always possible to avoid Equation (44) by raising the polynomial degrees of the discretization spaces [27, 28]. Here our argument is independent of the polynomial degree, hence locking always occurs using pure displacement-based finite element formulations. But of course, for a given thickness, the errors in the solution may be acceptably small if the order of the polynomials is sufficiently high and the mesh is sufficiently fine.

Much research has been performed to obtain locking-free finite element methods for shells, but only limited success has been achieved. For the simpler plate problern, mixed finite element methods are providing very efficient numerical schemes, leading to formulations of the type

$$
A\left(U_{t}^{h} ; V\right)+\frac{1}{t^{2}} D^{h}\left(U_{t}^{h} ; V\right)=F(V), \quad \forall V \in \mathcal{U}^{h},
$$

where $D^{h}$ is a perturbed form of $D$ (see Ref. [17] and the MITC elements in Refs $[1,29])$. In these methods, the locking-free behaviour is achieved as a consequence of the relaxation of the original constraint implied by the substitution of $D^{h}$ for $D$. The rigorous construction of the specific form of $D^{h}$ as well as the mathematical analysis of the finite element discretizations are based on a mathematical condition, known as the "inf-sup condition", which guarantees that the method is stable and optimal $[17,30]$. However, mixed finite element discretizations for shells are much more difficult to construct and analyse and so far the relevant infsup condition could not be proven to be satisfied by any discretization without resorting to over-simplifying assumptions on the geometry [31]. It seems that only more elaborate methods, known as mixed stabilized methods, which in fact circumvent the inf-sup condition, have allowed a complete mathematical analysis showing that locking is neutralized [32], but these procedures are more complex and, more seriously, lack generality of application (see next section).

\subsection{Inhibited shells}

In the inhibited case, we already mentioned the loss of regularity occurring in the asymptotic beha- viour. Obviously, we cannot expect reasonable numerical results when $t$ tends to zero unless the exact solution retains some degree of regularity. We therefore exclude from our consideration cases with critically ill-posed limit problems, such as an elliptic shell clamped on only part of its boundary. In such cases, we must probably accept to restrict the numerical computations to "reasonably small" values of $t$.

Assuming therefore that the solution retains some reasonable properties in the asymptotic limit, like in the example of an elliptic shell clamped along the whole boundary in the Koiter model, we can show that, under rather unrestrictive assumptions, any conforming finite element method satisfies a property of uniform convergence. Let $U_{t}^{h}$ be the finite element solution for the shell thickness $t$, i.e. $U_{t}^{h}$ satisfies

$$
D\left(U_{t}^{h} ; V\right)+t^{2} A\left(U_{t}^{h} ; V\right)=F(V), \quad \forall V \in \mathcal{U}^{h} .
$$

We denote by $\|\cdot\|_{\mathcal{U}}$ the norm used for $\mathcal{U}$ and define

$$
\|V\|_{\mathcal{V}} \stackrel{\text { def }}{=} D(V ; V)^{1 / 2}, \quad \forall V \in \mathcal{V} .
$$

Let $t_{\mathrm{s}}$ be an upper bound for the shell thickness to be considered, then we have the following proposition (which, considering practical experience, does not represent a surprise).

Proposition 5. We assume that $F \in \mathcal{V}$ and that there exist two interpolation operators $I^{h}$ and $J^{h}$, defined respectively on $\mathcal{V}$ and $\mathcal{U}$, both with values in $\mathcal{U}_{h}$, which satisfy the following properties:

$$
\left.\begin{array}{ll}
\forall V \in \mathcal{V}, & \left\|I^{h} V\right\|_{\mathcal{V}} \leq C_{1}\|V\|_{\mathcal{V}} \\
\forall V \in \mathcal{U}, & \left\|I^{h} V\right\|_{\mathcal{U}} \leq C_{2}\|V\|_{\mathcal{U}} \\
\forall V \in \mathcal{V}, & \lim _{h \rightarrow 0}\left\|V-I^{h} V\right\|_{\mathcal{V}}=0
\end{array}\right\}
$$

Then it holds that

$$
\lim _{h \rightarrow 0} \sup _{\left.t \in j 0, t_{s}\right]}\left\{\left\|U_{t}-U_{t}^{h}\right\|_{\mathcal{V}}+t\left\|U_{t}-U_{t}^{h}\right\|_{u}\right\}=0 .
$$

We give the proof in Appendix A.

This result ensures that the numerical approximations of Equation (13) remain uniformly convergent when $t$ tends to zero, which rules out any problem of the same type as locking. In order to obtain a more accurate estimate, for instance an order of uniform convergence (in $h$ ), we would need some properties of uniform regularity that are beyond our reach in the framework of the present paper.

We further point out that Equations (50) and (51) are rather unrestrictive assumptions. For instance, in the case of the uniformly elliptic Koiter shell clamped along the whole boundary, where we have $\mathcal{U}=\left[H_{0}^{1}(\Omega)\right]^{2} \times H_{0}^{2}(\Omega)$ and $\mathcal{V}=\left[H_{0}^{1}(\Omega)\right]^{2} \times L^{2}(\Omega)$, we can choose for both $I_{h}$ and $J_{h}$ the Clément in- 
terpolation operator [33] to fulfill the required assumptions.

\subsection{The general case}

In engineering practice, it is usually not expedient to determine to which category a particular shell belongs. Typically, a shell is defined as a thin threedimensional solid by a computer-aided design program and is not of uniform geometric nature. The structure may also contain some folds which are known to dramatically change the behaviour of a shell by increasing its stiffness. General finite element schemes that behave equally well for both inhibited and non-inhibited shells would thercfore be of utmost value.

For inhibited shells, we have seen that a direct conforming method is very likely to yield good results. For non-inhibited shells, however, these conforming methods are subject to locking and resorting to mixed methods is necessary. It is therefore interesting to study how these methods behave when applied to the inhibited case. We then solve a problem of the general form

$$
D^{h}\left(U_{t}^{h} ; V\right)+t^{2} A^{h}\left(U_{t}^{h} ; V\right)=F^{h}(V), \quad \forall V \in \mathcal{U}^{h} .
$$

where $D^{h}, A^{h}$ and $F^{h}$ are perturbed forms of $D, A$ and $F$ due to mixed interpolations. Note that, in the above discussions, we concentrated on the effect of the mixed interpolation on the $D$ form, so we assumed that $A^{h}=A$ and $F^{h}=F$. But this assumption is not necessary to observe that, whereas in the non-inhibited case the modification of $D$ can be interpreted as a relaxation of the constraint, in the inhibited case it perturbs the leading part of the variational formulation, as shown by the convergence result of Proposition 2. The key point is then whether or not this perturbation allows sufficient consistency between $D^{h}$ and $D$. If this consistency is not available, difficulties occur such as spurious modes or more generally speaking non-convergent solutions. For mixed stabilized methods, this question is even more acute as $D$ is more seriously perturbed than for a standard mixed method [32]. In the case $A^{h}$ and $F^{h}$ are not equal to $A$ and $F$, respectively, we of course also require consistency between $F^{h}$ and $F$, and (in the general case) between $A^{h}$ and $A$.

\section{NUMERICAL EVALUATION OF SHELL ANALYSIS PROCEDURES}

As we discussed in the previous sections, a particular difficulty in designing general finite element methods for shell structures lies in that an effective method should be equally well applicable to the bending-dominated situations in non-inhibited cases and the membrane-dominated situations in inhibited cases. Of course, the design of an effective shell element requires also a number of other consider- ations (specifically that the element must not contain a spurious energy mode) [1].

Since the mathematical analysis of most shell finite element discretizations used in practice is (still) out of reach, recourse must be taken to a numerical assessment of the convergence behaviour (which of course is also valuable even if a mathematical analysis is available). This assessment requires a study of the finite element scheme in judiciously selected test problems. The objective of the numerical tests is to precisely show whether a finite element scheme is a general procedure, i.e. is applicable to both categories of shell behaviours-the inhibited and non-inhibited cases-, and in how far the convergence rate is independent of the shell thickness. As previously mentioned, ideally the convergence rate for any shell geometry and boundary conditions is independent of the thickness $t$ and always optimal for the interpolations used. This objective is similar to the objective in almost incompressible analysis, where the convergence rate of a finite element scheme should be independent of the bulk modulus and optimal[1].

Of course, many test problems have been employed in shell finite element developments. In the following sections, we comment upon some widely-used problems and propose a sequence of test problems that we consider to be particularly suitable for the evaluation of shell finite elements.

\subsection{Remarks on some widely used benchmark pro- blems}

The three problems discussed below have been amply used as benchmark problems. While we point out some deficiencies in these problems, our remarks should, of course, not be interpreted as completely rejecting them as test problems.

4.1.1. The Scordelis-Lo roof. This problem is described in Fig. 7. The structure is subjected to its self-weight. Considering the boundary conditions, we directly infer from Section 2.5.3 that pure bending is inhibited. We show in Appendix B that the corresponding limit membrane problem is ill-posed due to the loading applied here.

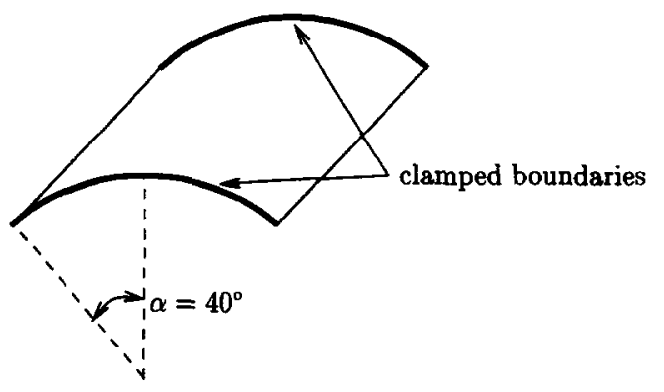

Fig. 7. Scordelis-Lo roof. 
4.1.2. The pinched cylinder. Figure 8 displays the reduced problem that results from symmetry considerations. The koundary conditions prescribed on the ends of the cylinder are $u_{2}=u_{3}=0$ (and $\theta_{1}=0$ for Reissner-Mindlin models). In this case, Equation (19) implies that pure bending is again inhibited. As regards the loading, whether or not the variational problem for $t$ finite is well-posed depends on the shell model used because a transverse concentrated load is associated with a pointwise transverse displacement through the variational principle. For a Kirchhoff-Love type model the admissible space for the transverse displacement is $H^{2}$ so that pointwise values can be considered. By contrast, for a Reissner-Mindlin type model, the transverse displacements are controlled in $H^{1}$ only, therefore pointwise values cannot be defined. Considering now the limit membrane problem, it is obviously ill-posed for both types of models since, as can be seen from the expression of the membrane strains, the transverse displacement can at most be measured in $L^{2}$.

4.1.3. The hemispherical shell. We consider the full hemispherical shell subjected to the concentrated loads showa in Fig. 9. The prescribed boundary conditions are the minimum required to prevent rigid body motions. Then pure bending is not inhibited [26], therefore the asymptotic behaviour is bending-dominated. Using the above argument, for $t$ finite the variational problem is well-posed for a Kirchhoff-Love type model, but ill-posed for a Reissner-Mindlin type model. The limit problem, however, is now posed in the subspace of inextensional displacements $\mathcal{U}_{0}$ which is more regular than $\mathcal{U}$. Hence the beriding limit problem is well-posed for both types of models since, in the ReissnerMindlin case, Equation (6) implies that a transverse displacement in $\mathcal{U}_{10}$ is also in $H^{2}$.

This problem may thus be used to test the behaviour of a finite element method for locking. However, as we mentioned in Section 2.5.1, the situation of a totally free boundary is the only way to obtain a bending-dominated behaviour with an

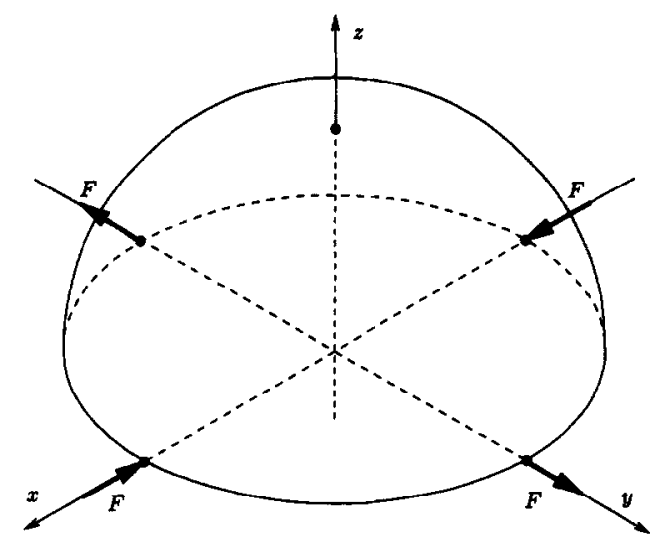

Fig. 9. Hemispherical shell.

elliptic surface, and any other boundary condition leads to membrane-dominated or strongly ill-posed problems. Because of the extreme sensitivity with respect to changes in the boundary conditions, we do not consider it a very suitable test problem for locking.

\subsection{Recommendations for a numerical evaluation strategy and suitable test problems}

It is clear from the conclusions of Section 3 that the design of a general finite element procedure for shell analysis must aim at overcoming two major difficulties: locking (in bending-dominated cases) must not occur and consistency in all terms must not be lost. In particular, there is a danger that, when removing membrane and shear locking, consistency is lost in the membrane term.

This suggests a natural strategy to numerically assess the reliability of a shell finite element procedure. A suitable sequence of test problems should fulfill the following requirements:

1. The test sequence should contain cases of both categories of asymptotic behaviours, namely bending-dominated and membrane-dominated cases.
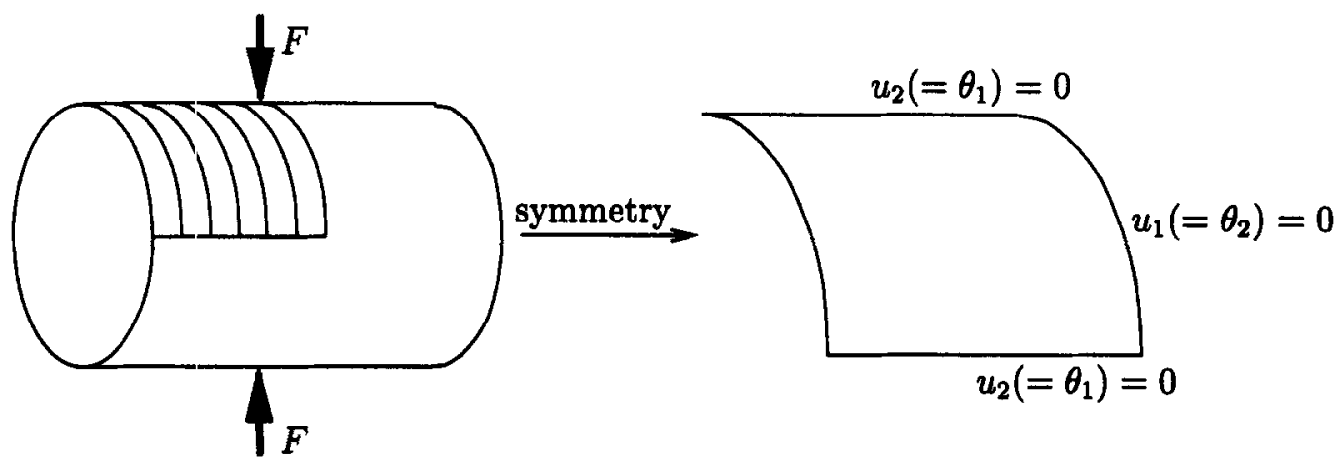

Fig. 8. Pinched cylinder and symmetry-reduced problem. 
2. Test cases corresponding to well-posed variational problems with well-posed limit problems should be preferably employed. Indeed, only well-posed problems allow a straightforward interpretation of any numerical difficulties.

3. For each category of asymptotic behaviour, several types of surface geometries-according to the elliptic/parabolic/hyperbolic classificationshould be considered. Since asymptotic behavionrs are highly sensitive to the geometry, this general testing is necessary in order to assure that a finite element scheme is a general procedure, and not one tuned to one particular type of test case.

Now that we defined a general evaluation strategy, we given some further recommendations on how the evaluation should be performed in order to allow a valuable interpretation of the solution results:

- Since we are primarily concerned with the detection of a possible deterioration of convergence of a given finite element procedure when the parameter $t$ decreases, it is crucial to compare convergence behaviours of the same discretization method for several values of $t$, for instance by measuring the errors obtained for a rather thick shell and a very thin one ( $t$ typically two orders of magnitude smaller).

- For membrane-dominated cases, an observed deterioration of convergence may be due to a loss of regularity of the exact solution rather than a loss of consistency of the finite element scheme.
This can be easily determined by comparing the results using the finite element procedure with the results obtained from a conforming displacementbased scheme. The conforming scheme, which should perform well according to Proposition 5, would only reflect the loss of regularity. Of course, all errors should be measured in a norm compatible with the membrane energy norm, since it is only in this norm that uniform convergence can be expected.

- In the construction of a finite element mesh, we should carefully avoid aligning the edges of the elements along curves featuring any particular geometric properties, such as asymptotic lines. Such alignment may alleviate locking in some instances [20], but would not be a general remedy for the analysis of shells with complex geometries (i.e. in industrial applications). These considerations are particularly important when using cylindrical shells in benchmark problems. Here, the natural tendency to align the mesh on the axis should be resisted.

We proceed to propose a sequence of suitable test cases which we divide according to their asymptotic behaviours.

4.2.1. Bending-dominated test problems. As bending-dominated test problems, we propose the following test cases:

- Full cylinder with free ends. The cylinder is loaded by a periodic pressure as shown in Fig. 10. No boundary conditions are prescribed, and rigid body modes are discarded by imposing appropri-

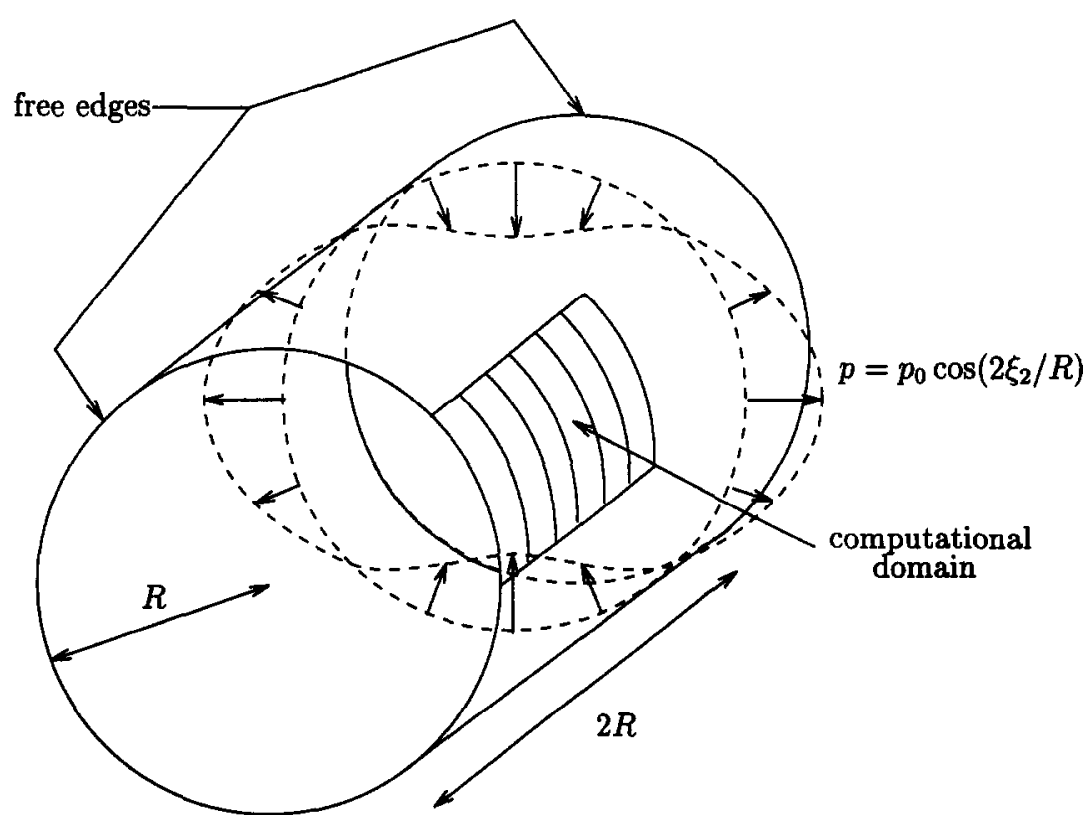

Fig. 10. Cylinder loaded by periodic pressure. 
ate symmetry conditions. Pure bending is clearly non-inhibited. This example was already proposed in Ref. [34], which also presented a procedure to obtain numerical solutions of arbitrary precision. In our experience it is an excellent test as regards locking [32].

- Partly-clamped hyperbolic paraboloid. The shell shown in Fig. 3 is clamped on one side and loaded by self weight. According to Section 2.5.2, pure bending is globally non-inhibited. We do not have an analytical solution for this problem but, for each value of $t$, the numerical solution obtained with the finest mesh can be used as a reference to plot error graphs. Of course, if the finite element method considered suffers from locking, this reference solution may not be accurate. However, a shift in the convergence plot thus-obtained as $t$ decreases indicates that the convergence is not uniform in $t$ and that therefore some locking is present, the severity of it depending on the amount of the shift. We advocate this test problem to detect locking because the geometry is more general than a surface of zero Gaussian curvature, and the problem is more realistic than the problem of an elliptic surface with free ends.

4.2.2. Membrane-dominated test problems. As membrane-dominated test problems, we propose the following test cases:

- Full cylinder with clamped ends. The geometric definition of this problem and the loading are the same as for the first proposed test above, but we now consider fully-clamped ends. The problem is also analysed in Ref. [34], where it is shown that pure bending is inhibited and that the membrane limit problem is well-posed. Here again, numerical solutions of arbitrary precision can be computed.

- Clamped hem spherical cap. We know from Section 2.4.2 that the space $\mathcal{V}$, on which the membrane problem is posed, is rather regular so that any reasonable distributed loading (in $L^{2}$, say) will provide a well-posed limit problem. If we choose an axisymmetric loading such as the one described in Fig. 11, we can compare the numerical results of the shell analysis procedure with the results of some reliable axisymmetric analysis scheme.

\section{CONCLUDING REMARKS}

The objective in this paper was to present and explain fundamental theoretical considerations regarding the analysis of shells, and discuss how to make use of these considerations for the development of improved general finite element analysis procedures. We review that shells, when the thick-

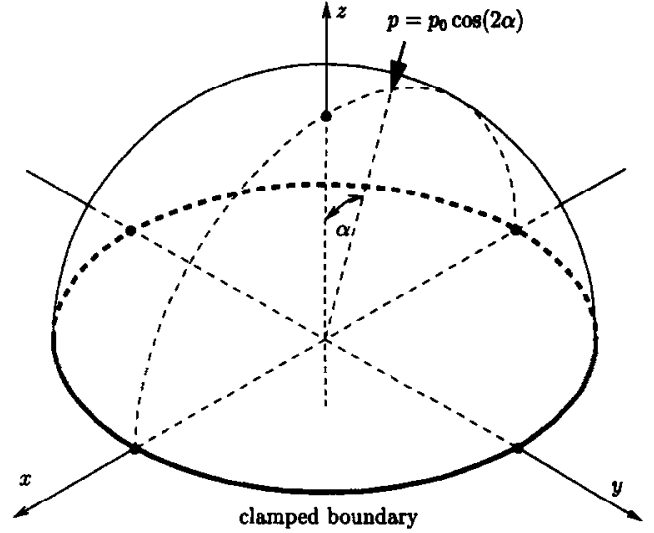

Fig. 11. Hemispherical shell under axisymmetric loading.

ness becomes small, fall into two dramatically different categories, namely bending-dominated and membrane-dominated structures. The design of a finite element procedure that is general and optimal for both cases is very difficult, and mathematical analyses of available finite element schemes hardly exist. Therefore, it is crucial to have available appropriate numerical test problems, and use these in a judicious manner to evaluate the capability of a finite element scheme. The theoretical presentation in this paper leads us to propose a test methodology and test problems that we believe to be valuable in the search for improved finite element procedures for shell structures.

Acknowledgements-D. Chapelle is indebted to Professor Michel Bernadou (INRIA and Pôle Universitaire Léonard de Vinci) and wishes to thank him for suggesting this area of investigation and for his continuous support while this work was performed. This author also acknowledges partial support from the Human Capital and Mobility Program "Shells: Mathematical Modeling and Analysis, Scientific Computing" of the Commission of the European Communities (Contract \# ERBCHRXCT940536).

\section{REFERENCES}

1. Bathe, K. J., Finite Element procedures. Prentice Hall, Englewood Cliffs, NJ, 1996.

2. Goldenweizer A. L., Theory of Elastic Thin Shells. Pergamon Press, Oxford, 1961.

3. Novozhilov, V. V., Thin Shell Theory. WoltersNoordhoff Publishing, Groningen, 1970.

4. Flügge, W., Stresses in Shells, 2nd edn. Springer, Berlin, 1973.

5. Ciarlet, P. G. and Lods, V. Analyse asymptotique des coques linéairement élastiques. I. Coques "membranaires". Comptes Rendus de l'Academie des Science, Paris, Série I, 1994, 318, 863-868.

6. Ciarlet, P. G., Lods, V. and Miara, B. Analyse asymptotique des coques linéairement élastiques. II. Coques "en flexion". Comptes Rendus de l'Academie des Science, Paris, Série I, 1994, 319, 95-100.

7. Piila, J. and Pitkäranta, J., Energy estimates relating different linear elastic models of a thin cylindrical shell. I. The membrane-dominated case. SIAM Journal of Mathematical Analysis, 1993, 24(1), 1-22. 
8. Piila, J. and Pitkäranta, J., Energy estimates relating different linear elastic models of a thin cylindrical shell. II: The case of free boundary. SIAM Journal of Mathematical Analysis, 1995, 26(4), 820-849.

9. Bernadou M., Finite Element Methods for Thin Shell Problems. John Wiley and Sons, New York, 1996.

10. Green A. E. and Zerna W., Theoretical Elasticity, 2nd edn. Oxford University Press, Oxford, 1968.

11. Naghdi P. M., Foundations of elastic shell theory. In Progress in Solid Mechanics, Vol. 4. North-Holland, Amsterdam, 1963, pp. 1-90.

12. Koiter, W. T., On the nonlinear theory of thin elastic shells. Koninklijke Nederlandse Akademie van Wetenschappen-Proceedings, 1965, B69, 1-54.

13. Bernadou M. and Ciarlet P. G., Sur l'ellipticité du modèle linéaire de coques de $W$. $T$. Koiter. In Computing Methods in Applied Sciences and Engineering, ed. R. Glowinski and J. L. Lions, Springer Verlag, Berlin. 1975.

14. Coutris, N., Théorème d'existence et d'unicité pour un problème de coque élastique dans le cas d'un modèle linéaire de P. M. Naghdi. RAIRO Analyse Numérique, 1978, 12, 51-57.

15. Sanchez-Palencia, E., Statique et dynamique des coques minces. I. Cas de flexion pure non inhibée. Comptes Rendus de l'Academie des Science, Paris, Série I, 1989, 309, 411-417.

16. Sanchez-Palencia, E. Statique et dynamique des coques minces. II. Cas de flexion pure inhibéeApproximation membranaire. Comptes Rendues Academie de Science, Paris, Serie I, 1989, 309, 531537.

17. Brezzi F. and Fortin M., Mixed and Hybrid Finite Element Methods. Springer, Berlin, 1991.

18. Bathe, K. J. and Brezzi F., On the convergence of a four-node plate bending element based on MindlinReissner plate theory and a mixed interpolation. In The Mathematics of Finite Elements and Applications $V$, ed. J. R. Whiteman. Academic Press, NY, 1985 , pp. 491-503.

19. Chenais, D. and Paumier, J.-C., On the locking phenomenon for a class of elliptic problems. Numerische Mathematik, 1994, 67, 427-440.

20. Pitkäranta, J., The problem of membrane locking in finite element analysis of cylindrical shells. Numerische Mathematik, 1992, 61, 523-542.

21. Sanchez-Palencia, E. Asymptotic and spectral properties of a class of singular-stiff problems. Journal de Mathématiques, Pures et Appliquées, 1992, 71, 379406.

22. Ciarlet, P. G. and Lods, V., Ellipticité des équations membranaires d'une coque uniformément elliptique. Comptes Rendus de l'Academie des Science, Paris, Série I, 1994, 318, 195-200.

23. Ciarlet, P. G. and Sanchez-Palencia, E., Un théorème d'existence et d'unicité pour les équations de coques membranaires. Comptes Rendus de l'Academie des Science, Paris, Série I, 1993, 317, 801-805.

24. Lions J. L., Perturbations Singulières dans les Problèmes aux Limites et en Contrôle Optimal. Springer, Berlin, 1973.

25. Sanchez-Palencia, E., Surfaces et coques élastiques mince: problèmes et défis. La Vie des Sciences, 1995, 12(3), 239-258.

26. Kirmse, A., Bending-dominated deformation of thin spherical shells: analysis and finite element approximation. SIAM Journal of Numerical Analysis, 1993, 30(4), 1015-1040.

27. Arnold, D. N., Discretization by finite elements of a model parameter dependent problem. Numerische Mathematik, 1981, 37, 405-421.
28. Paumier J. C., On the locking phenomenon for a linearly elastic clamped plate. Research Report 76, L.M.C., 1992.

29. Bathe, K. J., Bucalem, M. L. and Brezzi, F., Displacement and stress convergence of our MITC plate bending elements. Engineering Computations, 1990, 7(4), 291-302.

30. Iosilevich A., Bathe K. J. and Brezzi F., Numerical inf-sup analysis of MITC plate bending elements. Proceedings Symposium on Plates and Shells. Université Laval, July 1996.

31. Arnold, D. N. and Brezzi, F., Locking-free finite element methods for shells. Mathematics Computations, $1977,66(217), 1-14$.

32. Chapelle D. and Stenberg R., Stabilized finite element formulations for shells in a bending dominated state. Submitted to SIAM Journal of Numerical Analysis.

33. Clément, P., Approximation by finite element functions using local regularization. R.A.I.R.O., 1975, R-2, $77-84$.

34. Pitkäranta, J., Leino, Y., Ovaskainen, O. and Piila, J., Shell deformation states and the finite element method: a benchmark study of cylindrical shells. Computer Methods in Applied Mechanics and Engineering, 1995, 128, 81-121.

\section{APPENDIX A}

Proof of Proposition 5

In what follows, symbols $c$ and $C$ denote generic strictly positive constants, independent of both $t$ and $h$, which can have different values at different occurrences except when they appear with indices. Wc recall that formulation (13) is supposed to be elliptic for any $t>0$. In particular

$$
c\|V\|_{\mathcal{U}}^{2} \leq D(V ; V)+t_{\mathrm{s}}^{2} A(V ; V) \leq C\|V\|_{\mathcal{U}}^{2}, \quad \forall V \in \mathcal{U} \text {. (A1) }
$$

Hence, using

$$
D(V ; V)=\|V\|_{\mathcal{V}}^{2},
$$

we infer that, for any $t \in\left[0, t_{\mathrm{s}}\right]$, for any $V \in \mathcal{U}$,

$$
\begin{aligned}
c\left\{\|V\|_{\mathcal{V}}+t\|V\|_{\mathcal{U}}\right\}^{2} & \leq D(V ; V)+t^{2} A(V ; V) \\
& \leq C\left\{\|V\|_{\mathcal{V}}+t\|V\|_{\mathcal{U}}\right\}^{2} .
\end{aligned}
$$

Therefore, standard properties of conforming approximations entail

$$
\begin{gathered}
\left\|U_{t}-U_{t}^{h}\right\|_{\mathcal{V}}+t\left\|U_{t}-U_{t}^{h}\right\|_{\mathcal{U}} \leq C_{4} \inf _{V \in \mathcal{U}^{h}}\left\{\left\|U_{t}-V\right\|_{\mathcal{V}}\right. \\
\left.+t\left\|U_{t}-V\right\|_{\mathcal{U}}\right\} .
\end{gathered}
$$

Furthermore, we also obtain from eqn (Al) that

$$
r^{2}\|V\|_{\mathcal{U}}^{2} \leq C t^{2}[D(V ; V)+A(V ; V)], \quad \forall V \in \mathcal{U},
$$

so that, using Equation (16) and the fact (inferred from Proposition 2) that $\left\|U_{t}\right\|_{v}$ remains bounded, we get

$$
\lim _{t \rightarrow 0} t\left\|U_{i}\right\|_{\mathcal{U}}=0 \text {. }
$$

Consider now an arbitrary number $\varepsilon>0$. To prove Equation (52), we need to show that there exists $h_{\varepsilon}>0$ such that, $\left.\forall h \in] 0, h_{\varepsilon}\right]$,

$$
\sup _{\left.t \in] 0, t_{s}\right]}\left\{\left\|U_{t}-U_{t}^{h}\right\|_{\mathcal{V}}+t\left\|U_{t}-U_{t}^{h}\right\|_{U}\right\} \leq \varepsilon .
$$

From eqn (A5), there exists $\left.\left.t_{\varepsilon}>0, \forall t \in\right] 0, t_{\varepsilon}\right]$,

$$
C_{4}\left(1+C_{2}\right) t\left\|U_{t}\right\| u \leq \frac{\varepsilon}{2}
$$

which, combined with eqns (A3) and (50b), yields

$$
\sup _{t \in\left[0, t_{t}\right\}}\left\{\left\|U_{t}-U_{t}^{h}\right\|_{v}+t\left\|U_{t}-U_{t}^{h}\right\|_{u}\right\}
$$




$$
\begin{aligned}
& \leq \sup _{t \in\left[0, t_{\varepsilon}\right]} C_{4}\left\{\left\|U_{t}-I^{h} U_{t}\right\|_{\nu}+t\left\|U_{t}-I^{h} U_{t}\right\|_{U}\right\} \\
& \leq \sup _{\left.t \in] 0, t_{\varepsilon}\right]} C_{4}\left\{\left\|U_{t}-I^{h} U_{t}\right\|_{\mathcal{V}}+\left(1+C_{2}\right) t\left\|U_{t}\right\|_{U}\right\} \\
& \leq C_{4} \sup _{t \in\left[0, t_{t}\right]}\left\|U_{t}-I^{h} U_{t}\right\|_{\mathcal{V}}+\frac{\varepsilon}{2}
\end{aligned}
$$

The mapping $t \rightarrow U_{t}$ is clearly continuous from $] 0, t_{\mathrm{s}}$ ] into $\mathcal{U}$, hence into $\mathcal{V}$, and Proposition 2 shows that this extends to $\left[0, t_{\mathrm{s}}\right]$ for $\mathcal{V}$, provided that we let

$$
U_{t}(t=0) \stackrel{\text { def }}{=} U_{l}
$$

Therefore, Equation (50a) implies that the family of functions $\left(U_{t}-I^{h} U_{t}\right)_{h}$ is equicontinuous from $\left[0, t_{\mathrm{S}}\right]$ into $\mathcal{V}$. But for any $t \geqslant 0$, we have from Equation $(50 \mathrm{c})$

$$
\lim _{h \rightarrow 0}\left\|U_{t}-I^{h} U_{t}\right\|_{\nu}=0
$$

so we can invoke the Ascoli theorem to obtain

$$
\lim _{h \rightarrow 0} \sup _{t \in\left[0, t_{\varepsilon}\right]}\left\|U_{t}-I^{h} U_{t}\right\|_{\nu}=0 .
$$

It follows that there exists $h_{\varepsilon}^{(1)}>0$ such that, $\left.\left.\forall h \in\right] 0, h_{\varepsilon}^{(1)}\right]$,

$$
C_{4} \sup _{t \in\left[0, t_{\varepsilon}\right]}\left\|U_{t}-I^{h} U_{t}\right\|_{\mathcal{V}} \leq \frac{\varepsilon}{2}
$$

hence, from eqn (AB)

$$
\sup _{t \in\left\{0, I_{\varepsilon}\right]}\left\{\left\|U_{t}-U_{t}^{h}\right\|_{\mathcal{V}}+t\left\|U_{t}-U_{t}^{h}\right\|_{\mathcal{U}}\right\} \leq \varepsilon .
$$

Then, using eqn (A.3) we get

$$
\begin{aligned}
& \sup _{t \in\left[t_{t}, t_{s}\right]}\left\{\left\|U_{t}-U_{t}^{h}\right\|_{\nu}+t\left\|U_{t}-U_{t}^{h}\right\|_{U U}\right\} \\
& \leq \sup _{t \in\left[t_{\varepsilon}, t_{s}\right]} C_{4}\left\{\left\|U_{t}-J^{h} U_{t}\right\|_{\nu}+t\left\|U_{t}-J^{h} U_{t}\right\|_{u}\right\} \\
& \leq C_{5} \sup _{t \in\left\{t_{\varepsilon}, t_{s}\right\}}\left\|U_{t}-J^{h} U_{t}\right\|_{U} .
\end{aligned}
$$

From Equation (51a), we infer that the family $\left(U_{t}-J^{h} U_{t}\right)_{h}$ is equicontinuous from $\left[t_{\varepsilon}, t_{\mathrm{s}}\right]$ into $\mathcal{U}$. Therefore, Equation (51b) and the Ascoli theorem imply

$$
\lim _{h \rightarrow 0} \sup _{t \in\left[f_{t}, t_{3}\right]}\left\|U_{t}-J^{h} U_{t}\right\|_{\mathcal{U}}=0
$$

so that there exists $h_{\varepsilon}^{(2)}>0$ such that, $\left.\left.\forall h \in\right] 0, h_{\varepsilon}^{(2)}\right]$,

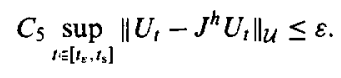

hence

$$
\sup _{t \in\left[L_{t}, t_{s}\right]}\left\{\left\|U_{t}-U_{t}^{h}\right\|_{v}+t\left\|U_{t}-U_{t}^{h}\right\|_{u}\right\} \leq \varepsilon .
$$

Now letting

$$
h_{\varepsilon} \stackrel{\text { def }}{=} \min \left(h_{\varepsilon}^{(1)}, h_{\varepsilon}^{(2)}\right)
$$

we get eqn (A6).

\section{APPENDIX B}

\section{Limit problem for the Scordelis-Lo roof}

In this appendix, we prove that the test case known as "Scordelis-Lo roof" is associated with an ill-posed membrane-dominated limit problem, by showing that Equation (15) does not hold. To this end, we construct a sequence of displacements $\left(V_{\varepsilon}\right)$ such that $D\left(V_{\varepsilon} ; V_{\varepsilon}\right)$ remains bounded, while $F\left(V_{\varepsilon}\right)$ tends to infinity when $\varepsilon$ tends to zero. For simplicity of computations, we assume that the structure is loaded by a uniform pressure denoted by $p$,

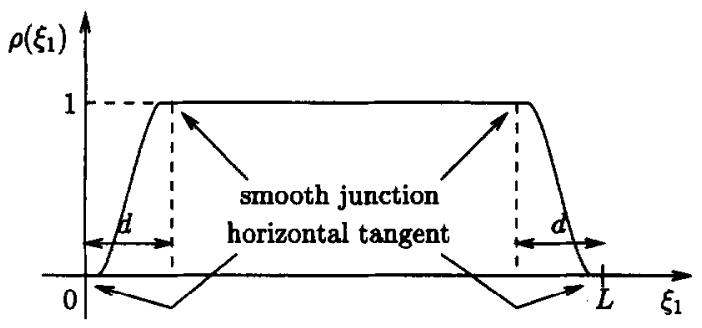

Fig. B1. Graphic definition of $\rho$.

but the same technique can be applied for a roof loaded with its self-weight and similar conclusions are reached. Thus we use

$$
F(V)=\int_{S} p v_{3} \mathrm{~d} S
$$

We recall that we consider clamped boundary conditions on the two extreme cross-sections (see Fig. 7).

We again use the natural coordinate system of the cylinder such as displayed in Fig. 5 . We start by noting that, for an arbitrary smooth function $\psi$, a displacement field $\mathrm{v}(\psi)$ defined by

$$
\left.\begin{array}{l}
v_{1}(\psi)=0 \\
v_{2}(\psi)=\psi\left(\xi_{2}\right) \\
v_{3}(\psi)=-R \psi^{\prime}\left(\xi_{2}\right)
\end{array}\right\}
$$

gives zero membrane strains according to System (19). Of course, except if $\psi$ is identically zero, this displacement field does not satisfy the clamped boundary conditions (we recall that $\mathcal{U}_{0}=\{0\}$ ). We then define

$$
\mathbf{w}(\psi) \stackrel{\text { def }}{=} \rho\left(\xi_{1}\right) \mathbf{v}(\psi),
$$

where $\rho$ is a smooth function of the type described in Fig. B1. This new displacement field satisfies the prescribed boundary conditions, and gives the following membrane strains

$$
\left.\begin{array}{l}
\gamma_{11}(\mathbf{w}(\psi))=0 \\
\gamma_{22}(\mathbf{w}(\psi))=0 \\
\gamma_{12}(\mathbf{w}(\psi))=\frac{1}{2} \rho^{\prime}\left(\xi_{1}\right) \psi\left(\xi_{2}\right)
\end{array}\right\}
$$

We now consider a sequence of functions $\left(\psi_{\varepsilon}\right)$ defined as follows (see also Fig. B2)

$$
\begin{aligned}
\psi_{\varepsilon}\left(\xi_{2}\right) & =\frac{1}{\sqrt{\varepsilon}}\left(1-\frac{\xi_{2}}{\varepsilon}\right)^{3} \text { for } 0 \leq \xi_{2} \leq \varepsilon \\
& =0 \text { for } \varepsilon \leq \xi_{2}
\end{aligned}
$$

and we construct a displacement sequence $\left(V_{\varepsilon}\right)$ associated with $\left(w\left(\psi_{s}\right)\right)$. If the model considered is of the ReissnerMindlin type, we choose the rotations so that the shear strains are zero (cf. Equation (6)). This is allowed since $w\left(\psi_{\varepsilon}\right)$ ) is sufficiently smooth (in particular, $\psi_{\varepsilon}$ is twice continuously differentiable at $\xi_{2}=\varepsilon$ ). Then we have

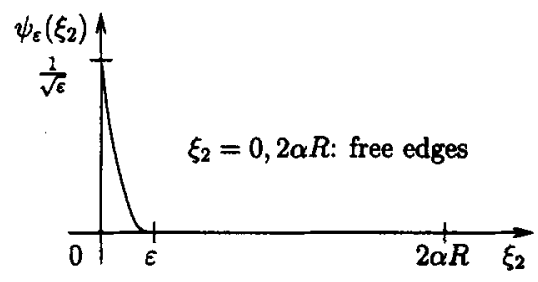

Fig. B2. Plot of $\psi_{\epsilon}$. 


$$
\begin{aligned}
D\left(V_{\varepsilon} ; V_{\varepsilon}\right) & \propto \int_{S}\left[\gamma_{12}\left(\mathbf{w}\left(\psi_{\varepsilon}\right)\right)\right]^{2} \mathrm{~d} S \\
& \propto \int_{S}\left[\psi_{\varepsilon}\left(\xi_{2}\right)\right]^{2} \mathrm{~d} S \\
& \propto\left(\frac{1}{\sqrt{\varepsilon}}\right)^{2} \times \varepsilon=1
\end{aligned}
$$

Therefore, Condition (15) is violated and Problem (14) is ill-posed. In other words, the membrane deformation energy is unable to appropriately control some specific displacement fields that are excited by the loading, such as the sequence of displacements that we constructed. This suggests that strong singularities will appear near the free boundary when the thickness tends to zero.

whereas, from Equation (69)

$$
\begin{aligned}
F\left(V_{\varepsilon}\right)= & \int_{S} p w_{3}\left(\psi_{\varepsilon}\right) \mathrm{d} S \\
= & -\int_{\xi_{1}, \xi_{2}} p R \rho\left(\xi_{1}\right) \psi_{\varepsilon}^{\prime}\left(\xi_{2}\right) \mathrm{d} \xi_{1} \mathrm{~d} \xi_{2} \\
= & -\int_{\xi_{1}}\left\{p R \rho\left(\xi_{1}\right) \psi_{\varepsilon}\left(\xi_{2}\right)\right]_{\xi_{2}=0}^{2 \alpha R} \\
& \left.-\int_{\xi_{2}} p R \frac{\partial \rho\left(\xi_{1}\right)}{\partial \xi_{2}} \psi_{\varepsilon}\left(\xi_{2}\right) \mathrm{d} \xi_{2}\right\} \mathrm{d} \xi_{1} \\
= & p R \psi_{\varepsilon}(0) \int_{\xi_{1}} \rho\left(\xi_{1}\right) \mathrm{d} \xi_{1} \\
\propto & \frac{1}{\sqrt{\varepsilon}}
\end{aligned}
$$

